\title{
Denumerably many positive solutions for a $n$-dimensional higher-order singular fractional differential system
}

Ping Li and Meiqiang Feng*
*Correspondence:

meiqiangfeng@sina.com

School of Applied Science, Beijing University, Beijing, People's Republic of China Information Science \& Technology

\section{Springer}

Keywords: Denumerably many positive solutions; $n$-dimensional higher-order fractional differential system; Infinitely many singularities; Matrix theory; Fixed point theorems in cones

\begin{abstract}
This paper investigates the existence of denumerably many positive solutions and two infinite families of positive solutions for the $n$-dimensional higher-order fractional differential system $\mathbf{D}_{0^{+}}^{\alpha} \mathbf{x}(t)+\lambda \mathbf{g}(t) \mathbf{f}(t, \mathbf{x}(t))=0,0<t<1$. The vector-valued function $\mathbf{x}$ is defined by $\mathbf{x}=\left[x_{1}, x_{2}, \ldots, x_{n}\right]^{\top}, \mathbf{g}(t)=\operatorname{diag}\left[g_{1}(t), g_{2}(t), \ldots, g_{n}(t)\right]$, where $g_{i} \in L^{p}[0,1]$ for some $p \geq 1, i=1,2, \ldots, n$, and has infinitely many singularities in $\left[0, \frac{1}{2}\right)$. Our methods employ the fixed point theorems combined with the partially ordered structure of a Banach space.

\section{Abstract}

theorems in cones (

\section{Introduction}

Fractional differential equations, which provide a natural description of memory and hereditary properties of various materials and processes, are regarded as an important mathematical tool for better understanding of many real world problems in applied sciences, such as physics, chemistry, aerodynamics, Bode's analysis of feedback amplifiers, capacitor theory, electrical circuits and so on. This is the main advantage of fractional differential equations in comparison with classical integer-order models. For applications and explanations of fractional differential equations, we refer the reader to the texts [1-4]. In particular, many authors have shown great interest in the subject of fractional-order boundary value problems (BVPs), and many excellent results for BVPs equipped with different kinds of boundary conditions have been obtained, for more details and examples, see [5-23] and the references cited therein.

Moreover, Alsulami, Ntouyas, Agarwal, Ahmad and Alsaedi [24] pointed out that fractional differential system constitute an important and interesting field of investigation because of their applications in many real world problems such as anomalous diffusion [25], disease models [26-28], ecological models [29], synchronization of chaotic systems [30-32] and so forth. For some theoretical work on the fractional differential system, we refer the reader to [33-41]. However, it is not difficult to see that there are only a few results on $n$-dimensional fractional differential systems; for example, see

(c) The Author(s) 2018. This article is distributed under the terms of the Creative Commons Attribution 4.0 International License (http://creativecommons.org/licenses/by/4.0/), which permits unrestricted use, distribution, and reproduction in any medium, provided you give appropriate credit to the original author(s) and the source, provide a link to the Creative Commons license, and indicate if changes were made. 
[42, 43], especially for $n$-dimensional higher-order singular fractional differential systems.

At the same time, we notice that a class of boundary value problem with integral boundary conditions have attracted the attention of Boucherif [44], Zhang et al. [45, 46], Hao et al. [47], Jiang et al. [48, 49], Kong [50], Feng et al. [51], Ahmad et al. [52], Mao et al. [53], and Liu et al. $[54,55]$.

To the best of our knowledge, in the literature there are not articles on denumerably many positive solutions for the analogous of $n$-dimensional higher-order singular fractional differential with integral boundary conditions. More precisely, the study of $g_{i} \in L^{p}[0,1]$ for some $p \geq 1, i=1,2, \ldots, n$, and having infinitely many singularities in $\left[0, \frac{1}{2}\right)$, is still open for the following $n$-dimensional higher-order singular fractional differential system:

$$
\mathbf{D}_{0^{+}}^{\alpha} \mathbf{x}(t)+\lambda \mathbf{g}(t) \mathbf{f}(t, \mathbf{x}(t))=0, \quad 0<t<1
$$

with the following boundary conditions:

$$
\left\{\begin{array}{l}
\mathbf{x}(0)=\mathbf{x}^{\prime}(0)=\cdots=\mathbf{x}^{(n-2)}(0)=0 \\
a \mathbf{x}(1)+b \mathbf{x}^{\prime}(1)=\int_{0}^{1} \mathbf{h}(t) \mathbf{x}(t) d t
\end{array}\right.
$$

where $\mathbf{D}_{0+}^{\alpha}$ is the standard Riemann-Liouville fractional derivative of order $n-1<\alpha \leq n$, $n \geq 3, \lambda$ is a positive parameter, $a>0, b \geq 0$ and $a>(\alpha-1) b$. In addition,

$$
\begin{aligned}
& \mathbf{x}=\left[x_{1}, x_{2}, \ldots, x_{n}\right]^{\top}, \\
& \mathbf{g}(t)=\operatorname{diag}\left[g_{1}(t), g_{2}(t), \ldots, g_{n}(t)\right], \\
& \mathbf{f}(t, \mathbf{x})=\left[f_{1}(t, \mathbf{x}), f_{2}(t, \mathbf{x}), \ldots, f_{i}(t, \mathbf{x}), \ldots, f_{n}(t, \mathbf{x})\right]^{\top}, \\
& \mathbf{h}(t)=\operatorname{diag}\left[h_{1}(t), h_{2}(t), \ldots, h_{n}(t)\right],
\end{aligned}
$$

where

$$
f_{i}(t, \mathbf{x})=f_{i}\left(t, x_{1}, x_{2}, \ldots, x_{i}, \ldots, x_{n}\right)
$$

Therefore, system (1.1) means that

$$
\begin{cases}-\mathbf{D}_{0^{+}}^{\alpha} x_{1}(t)=\lambda g_{1}(t) f_{1}\left(t, x_{1}(t), x_{2}(t), \ldots, x_{n}(t)\right), & 0<t<1, \\ -\mathbf{D}_{0^{+}}^{\alpha} x_{2}(t)=\lambda g_{2}(t) f_{2}\left(t, x_{1}(t), x_{2}(t), \ldots, x_{n}(t)\right), & 0<t<1, \\ \ldots & \cdots, \\ -\mathbf{D}_{0^{+}}^{\alpha} x_{n}(t)=\lambda g_{n}(t) f_{n}\left(t, x_{1}(t), x_{2}(t), \ldots, x_{n}(t)\right), & 0<t<1,\end{cases}
$$


(1.2) means that

$$
\left\{\begin{array}{l}
x_{1}^{\prime}(0)=x_{1}^{\prime}(1)=\cdots=x_{1}^{(n-2)}(t)=0, \\
a x_{1}(1)+b x_{1}^{\prime}(1)=\int_{0}^{1} h_{1}(t) x_{1}(t) d t, \\
x_{2}^{\prime}(0)=x_{2}^{\prime}(1)=\cdots=x_{2}^{(n-2)}(t)=0, \\
a x_{2}(1)+b x_{2}^{\prime}(1)=\int_{0}^{1} h_{2}(t) x_{2}(t) d t, \\
\cdots, \\
x_{n}^{\prime}(0)=x_{n}^{\prime}(1)=\cdots=x_{n}^{(n-2)}(t)=0, \\
a x_{n}(1)+b x_{n}^{\prime}(1)=\int_{0}^{1} h_{n}(t) x_{n}(t) d t .
\end{array}\right.
$$

Here we emphasize that our problem is new in the sense of the $n$-dimensional higherorder singular fractional differential systems introduced here. To the best of our knowledge, the existence of single or multiple positive solutions for $n$-dimensional higher-order singular fractional differential system (1.1)-(1.2) has not yet to be studied, especially for the existence of denumerably many positive solutions and two infinite families of positive solutions for system (1.1)-(1.2). In consequence, our main results of the present work will be a useful contribution to the existing literature on the topic of $n$-dimensional higherorder singular fractional differential systems. The existence of denumerably many positive solutions and two infinite families of positive solutions for the given problem are new, though they are proved by applying the well-known method based on the fixed theory in cones and the partially ordered structure of Banach space.

Throughout this paper, we use $i=1,2, \ldots, n$, unless otherwise stated.

Let the components of $\mathbf{g}$ and $\mathbf{f}$ satisfy the following conditions:

$\left(H_{1}\right) g_{i} \in L^{p}[0,1]$ for some $p \in[1,+\infty)$, and there exists $N_{i}>0$ such that $g_{i}(t) \geq N_{i}$ a.e. on $J=[0,1]$;

$\left(H_{2}\right)$ there exists a sequence $\left\{t_{j}^{\prime}\right\}_{j=1}^{\infty}$ such that $t_{1}^{\prime}<\frac{1}{2}, t_{j}^{\prime} \downarrow t_{0}^{\prime}>0$ and $\lim _{t \rightarrow t_{j}^{\prime}} g_{i}(t)=+\infty$ for all $j=1,2, \ldots$;

$\left(H_{3}\right) f_{i}(t, \mathbf{x}) \in C\left(J \times R_{+}^{n}, R_{+}\right)$, where $R^{+}=[0,+\infty)$ and $R_{+}^{n}=\prod_{i=1}^{n} R_{+}$;

$\left(H_{4}\right) h_{i} \in L^{1}[0,1]$ is nonnegative with $\mu_{i} \in[0, a-(\alpha-1) b)$, where $\mu_{i}$ is defined in (2.18).

The plan of this paper is as follows. We shall introduce some basic definitions and lemmas of fractional calculus in the rest of this section. In Sect. 2, we give the expression and new properties of Green's function associated with system (1.1)-(1.2). In Sect. 3, we present some characteristics of the integral operator associated with system (1.1)-(1.2) and state two fixed point theorems in cones. In Sect. 4, we discuss the existence of denumerably many positive solutions of system (1.1)-(1.2). In Sect. 5, we will prove the existence of two infinite families of positive solutions of system (1.1)-(1.2). In Sect. 6, we give some interesting comments and remarks associated with system (1.1)-(1.2).

In the rest of this section, we introduce some basic definitions and lemmas of fractional calculus.

Definition $1.1([2])$ The integral

$$
I_{0+}^{\alpha} f(x)=\frac{1}{\Gamma(\alpha)} \int_{0}^{x} \frac{f(t)}{(x-t)^{1-\alpha}} d t, \quad x>0,
$$

where $\alpha>0$, is called Riemann-Liouville fractional integral of order $\alpha$. 
Definition $1.2([2])$ For a function $f(x)$ given in the interval $[0,1)$, the expression

$$
D_{0+}^{\alpha} f(x)=\frac{1}{\Gamma(n-\alpha)}\left(\frac{d}{d x}\right)^{n} \int_{0}^{x} \frac{f(t)}{(x-t)^{\alpha-n+1}} d t
$$

where $n=[\alpha]+1,[\alpha]$ denotes the integer part of number $\alpha$, is called the Riemann-Liouville fractional derivative of order $\alpha$.

Lemma 1.1 ([7]) Assume that $u \in C(0,1) \cap L(0,1)$ with a fractional derivative of order $\alpha>0$ that belongs to $u \in C(0,1) \cap L(0,1)$. Then

$$
I_{0+}^{\alpha} D_{0+}^{\alpha} u(t)=u(t)+C_{1} t^{\alpha-1}+C_{2} t^{\alpha-2}+\cdots++C_{N} t^{\alpha-N},
$$

for some $C_{i} \in R, i=1,2, \ldots, N$, where $N$ is the smallest integer greater than or equal to $\alpha$.

\section{Green's function associated with system (1.1)-(1.2)}

In this section, we discuss the expression and properties of the Green's function associated with system (1.1)-(1.2).

Let $\mathbf{y}=\left[y_{1}, y_{2}, \ldots, y_{n}\right]^{\top}$. Consider the fractional differential system

$$
\mathbf{D}_{0^{+}}^{\alpha} \mathbf{x}(t)+\mathbf{y}(t)=0, \quad 0<t<1,
$$

with the boundary conditions (1.2). Equation (2.1) means that

$$
\begin{cases}-\mathbf{D}_{0^{+}}^{\alpha} x_{1}(t)=y_{1}(t), & 0<t<1, \\ -\mathbf{D}_{0^{+}}^{\alpha} x_{2}(t)=y_{2}(t), & 0<t<1, \\ \cdots & \cdots, \\ -\mathbf{D}_{0^{+}}^{\alpha} x_{n}(t)=y_{n}(t), & 0<t<1 .\end{cases}
$$

Lemma 2.1 If $\int_{0}^{1} h_{i}(t) t^{\alpha-1} d t \neq a-(\alpha-1) b$ and $y_{i} \in C[0,1], i=1,2, \ldots, n$, then system (2.1)(1.2) has a unique solution $\mathbf{x}=\left[x_{1}, x_{2}, \ldots, x_{n}\right]^{\top} \in R_{+}^{n}$ in which $x_{i}(t)$ is given by

$$
x_{i}(t)=\int_{0}^{1} G_{i}(t, s) y_{i}(s) d s
$$

where

$$
\begin{aligned}
& G_{i}(t, s)=G_{1}(t, s)+G_{2 i}(t, s), \\
& G_{1}(t, s)=\frac{1}{\Gamma(\alpha)(a+(\alpha-1) b)}\left\{\begin{array}{c}
a t^{\alpha-1}(1-s)^{\alpha-1}+b(\alpha-1) t^{\alpha-1}(1-s)^{\alpha-2} \\
-(a+(\alpha-1) b)(t-s)^{\alpha-1}, \quad 0 \leq s \leq t \leq 1, \\
a t^{\alpha-1}(1-s)^{\alpha-1}+b(\alpha-1) t^{\alpha-1}(1-s)^{\alpha-2}, \\
0 \leq t \leq s \leq 1,
\end{array}\right. \\
& G_{2 i}(t, s)=\frac{t^{\alpha-1}}{(a-(\alpha-1) b)-\int_{0}^{1} h_{i}(t) t^{\alpha-1} d t} \int_{0}^{1} h_{i}(t) G_{1}(t, s) d t .
\end{aligned}
$$


Proof By the fact that system (2.1)-(1.2) is equivalent to system (2.2)-(1.4). Therefore system (2.1)-(1.2) has a unique solution $\mathbf{x}$ which is equivalent to the following system:

$$
\left\{\begin{array}{l}
\mathbf{D}_{0+}^{\alpha} x_{i}(t)+y_{i}(t)=0, \quad 0<t<1, \\
x_{i}(0)=x_{i}^{\prime}(0)=\cdots=x_{i}^{(n-2)}(0)=0 \\
a x_{i}(1)+b x_{i}^{\prime}(1)=\int_{0}^{1} h_{i}(t) x_{i}(t) d t
\end{array}\right.
$$

has a unique solution $x_{i}$, which is given by (2.3).

Next, by a proof which is similar to that of Lemma 2.1 in [8], we can show that (2.3) holds. This finishes the proof of Lemma 2.1.

From (2.4), (2.5) and (2.6), we can prove that $G_{i}(t, s), G_{1}(t, s)$ and $G_{2 i}(t, s)$ have the following properties.

Proposition 2.1 The function $G_{1}(t, s)$ defined by (2.5) satisfies

(i) $G_{1}(t, s) \geq 0$ is continuous for all $t, s \in J, G_{1}(t, s)>0, \forall t, s \in(0,1)$;

(ii) For all $t \in J, s \in(0,1)$, we have

$$
\begin{aligned}
G_{1}(t, s) \leq & G_{1}(\tau(s), s) \\
= & \left(a(\tau(s))^{\alpha-1}(1-s)^{\alpha-1}+b(\alpha-1)(\tau(s))^{\alpha-1}(1-s)^{\alpha-2}\right. \\
& \left.-(a+b(\alpha-1))(\tau(s)-s)^{\alpha-1}\right) /(\Gamma(\alpha)(a+b(\alpha-1))),
\end{aligned}
$$

where

$$
\tau(s)=\frac{s}{1-e(s)(1-s)^{\frac{\alpha-1}{\alpha-2}}}, \quad e(s)=\left[\frac{a+\frac{b(\alpha-1)}{1-s}}{a+b(\alpha-1)}\right]^{\frac{1}{\alpha-2}} .
$$

Proof (i) It is obvious that $G_{1}(t, s)$ is continuous on $J \times J$ and $G_{1}(t, s) \geq 0$ when $s \geq t$.

For $0 \leq s<t \leq 1$, we have

$$
\begin{aligned}
& a t^{\alpha-1}(1-s)^{\alpha-1}+b(\alpha-1) t^{\alpha-1}(1-s)^{\alpha-2}-(a+(\alpha-1) b)(t-s)^{\alpha-1} \\
& \quad=(1-s)^{\alpha-1}\left[\left(a+b(\alpha-1)(1-s)^{-1}\right) t^{\alpha-1}-(a+b(\alpha-1))\left(\frac{t-s}{1-s}\right)^{\alpha-1}\right] \\
& \quad \geq 0
\end{aligned}
$$

So, by (2.5), we have

$$
G_{1}(t, s) \geq 0, \quad \forall t, s \in J
$$

Similarly, for $t, s \in(0,1)$, we have $G_{1}(t, s)>0$. 
(ii) Since $n-1<\alpha \leq n, n \geq 3$, it is clear that $G_{1}(t, s)$ is increasing with respect to $t$ for $0 \leq t \leq s \leq 1$.

On the other hand, from the definition of $G_{1}(t, s)$, for given $s \in(0,1), s<t \leq 1$, we have

$$
\begin{aligned}
\frac{\partial G_{1}(t, s)}{\partial t}= & \frac{\alpha-1}{\Gamma(\alpha)(a+b(\alpha-1))}\left\{a t^{\alpha-2}(1-s)^{\alpha-1}+b(\alpha-1) t^{\alpha-2}(1-s)^{\alpha-2}\right. \\
& \left.-[a+b(\alpha-1)](t-s)^{\alpha-2}\right\} .
\end{aligned}
$$

Let

$$
\frac{\partial G_{1}(t, s)}{\partial t}=0
$$

Then we have

$$
a t^{\alpha-2}(1-s)^{\alpha-1}+b(\alpha-1) t^{\alpha-2}(1-s)^{\alpha-2}=[a+b(\alpha-1)](t-s)^{\alpha-2},
$$

and so

$$
\left(a+\frac{b(\alpha-1)}{1-s}\right)(1-s)^{\alpha-1}=[a+b(\alpha-1)]\left(1-\frac{s}{t}\right)^{\alpha-2} .
$$

Noticing $\alpha>2$, from (2.10), we have

$$
t=\frac{s}{1-e(s)(1-s)^{\frac{\alpha-1}{\alpha-2}}}=: \tau(s), \quad e(s)=\left[\frac{a+\frac{b(\alpha-1)}{1-s}}{a+b(\alpha-1)}\right]^{\frac{1}{\alpha-2}}
$$

Then, for given $s \in(0,1)$, we have $G_{1}(t, s)$ arriving at a maximum at $(\tau(s), s)$ when $s<t$. From this, together with the fact that $G_{1}(t, s)$ is increasing on $s \geq t$, we see that (2.8) holds.

Remark 2.1 From Fig. 1, we can see $G_{1}(s, s) \leq G_{1}(\tau(s), s)$ for $\alpha>2$. If $1<\alpha \leq 2$, then

$$
G_{1}(t, s) \leq G_{1}(s, s)=\frac{a s^{\alpha-1}(1-s)^{\alpha-1}+b(\alpha-1) s^{\alpha-1}(1-s)^{\alpha-2}}{\Gamma(\alpha)(a+b(\alpha-1))} .
$$

Figure 1 Graph of function $G_{1}(\tau(s), s), G_{1}(s, s)$ for $\alpha=5 / 2, a=1, b=0$

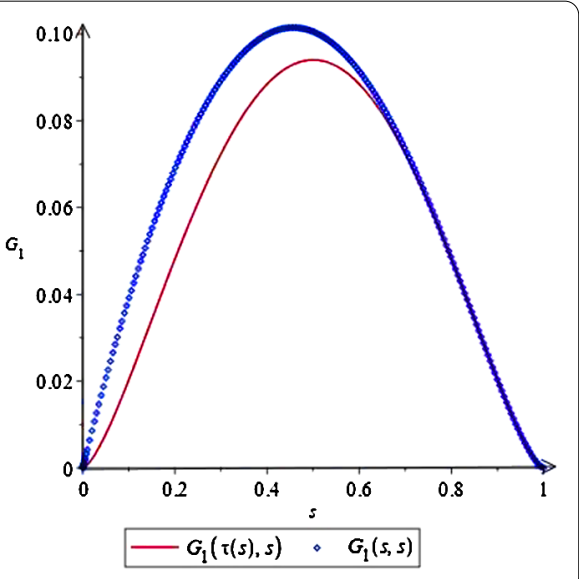



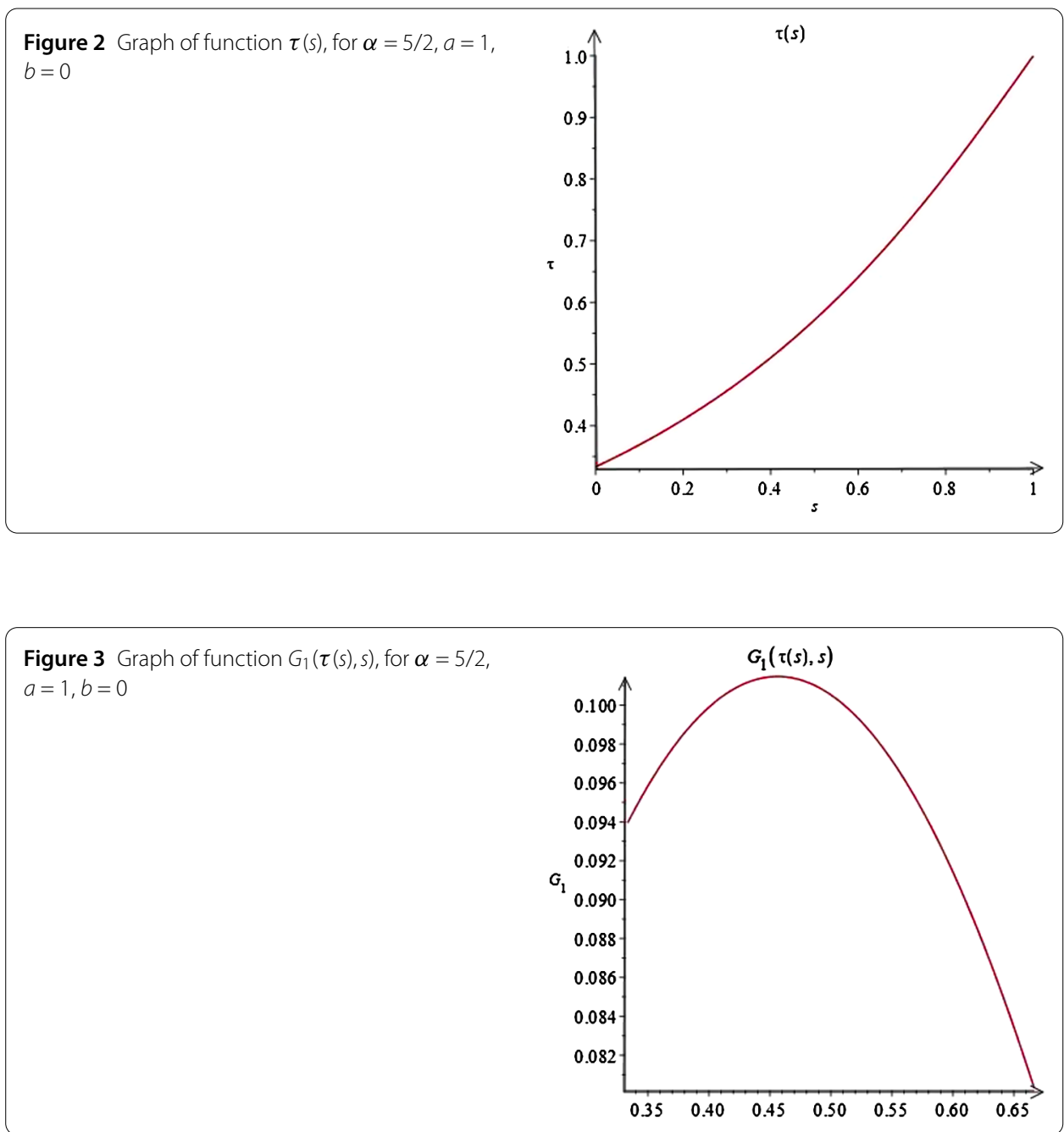

Remark 2.2 From Fig. 2, we can see that $\tau(s)$ is increasing with respect to $s$.

Remark 2.3 From Fig. 3, we can see that $G_{1}(\tau(s), s)>0$ for $s \in J_{\theta}=[\theta, 1-\theta]$, where $\theta \in$ $\left(0, \frac{1}{2}\right)$.

Remark 2.4 Let $\bar{G}_{1}(\tau(s), s)=a(\tau(s))^{\alpha-1}(1-s)^{\alpha-1}+b(\alpha-1)(\tau(s))^{\alpha-1}(1-s)^{\alpha-2}-(a+b(\alpha-$ $1))(\tau(s)-s)^{\alpha-1}$. From (2.8), for $s \in(0,1)$, we have

$$
\begin{aligned}
\frac{d \bar{G}_{1}(\tau(s), s)}{d s}= & -a(\alpha-1)(1-s)^{\alpha-2}(\tau(s))^{\alpha-1} \\
& +a(\alpha-1)(1-s)^{\alpha-1}(\tau(s))^{\alpha-1}\left(\tau^{\prime}(s)\right) \\
& -b(\alpha-1)(\alpha-2)(1-s)^{\alpha-3}(\tau(s))^{\alpha-1} \\
& +b(\alpha-1)^{2}(1-s)^{\alpha-2}(\tau(s))^{\alpha-2}\left(\tau^{\prime}(s)\right) \\
& -(\alpha-1)(\alpha-1)(a+b(\alpha-1))(\tau(s)-s)^{\alpha-2}\left(\tau^{\prime}(s)-1\right)
\end{aligned}
$$


Remark 2.5 From (2.11), we have

$$
\begin{aligned}
\lim _{s \rightarrow 0} \frac{d \bar{G}_{1}(\tau(s), s)}{d s} & =(\alpha-1)\left[-(a+b(\alpha-2))\left(\frac{\alpha-2}{\alpha-1}\right)^{\alpha-1}+(a+b(\alpha-1))\left(\frac{\alpha-2}{\alpha-1}\right)^{\alpha-2}\right] \\
& =\left(\frac{\alpha-2}{\alpha-1}\right)^{\alpha-2}(a+b(2 \alpha-3)) \\
& :=f(\alpha) .
\end{aligned}
$$

Remark 2.6 Noticing that $\alpha>2$, it follows from Remark 2.5 that $f(\alpha)>0$.

Remark 2.7 It is interesting to point out that $f(\alpha)$ is not decreasing with respect to $\alpha$ in the case with $a>0$ and $b>0$. If $a>0$ and $b=0$, then $f(\alpha)$ is decreasing with respect to $\alpha$.

Proposition 2.2 There exists $\gamma>0$ such that

$$
\min _{t \in[\theta, 1-\theta]} G_{1}(t, s) \geq \gamma G_{1}(\tau(s), s), \quad \forall s \in J
$$

Proof For $t \in J_{\theta}$, we divide the proof into the following three cases for $s \in J$.

Case 1. If $s \in J_{\theta}$, then from (i) of Proposition 2.1 and Remark 2.3, we have

$$
G_{1}(t, s)>0, \quad G_{1}(\tau(s), s)>0, \quad \forall t, s \in J_{\theta} .
$$

It is obvious that $G_{1}(t, s)$ and $G_{1}(\tau(s), s)$ are bounded on $J_{\theta}$. So, there exists a constant $\gamma_{1}>0$ such that

$$
G_{1}(t, s) \geq \gamma_{1} G_{1}(\tau(s), s), \quad \forall t, s \in J_{\theta}
$$

Case 2. If $s \in[1-\theta, 1]$, then from (2.5), we have

$$
G_{1}(t, s)=\frac{a t^{\alpha-1}(1-s)^{\alpha-1}+b(\alpha-1) t^{\alpha-1}(1-s)^{\alpha-2}}{\Gamma(\alpha)(a+b(\alpha-1))} .
$$

On the other hand, from the definition of $\tau(s)$, we see that $\tau(s)$ takes its maximum 1 at $s=1$. So

$$
\begin{aligned}
G_{1}(\tau(s), s)= & \left(a(\tau(s))^{\alpha-1}(1-s)^{\alpha-1}+b(\alpha-1)(\tau(s))^{\alpha-1}(1-s)^{\alpha-2}\right. \\
& \left.-(a+b(\alpha-1))(\tau(s)-s)^{\alpha-1}\right) /(\Gamma(\alpha)(a+b(\alpha-1))) \\
\leq & \frac{a(\tau(s))^{\alpha-1}(1-s)^{\alpha-1}+b(\alpha-1)(\tau(s))^{\alpha-1}(1-s)^{\alpha-2}}{\Gamma(\alpha)(a+b(\alpha-1))} \\
= & \frac{(\tau(s))^{\alpha-1}}{t^{\alpha-1}} \frac{a(1-s)^{\alpha-1} t^{\alpha-1}+b(\alpha-1)(1-s)^{\alpha-2} t^{\alpha-1}}{\Gamma(\alpha)(a+b(\alpha-1))} \\
\leq & \frac{1}{\theta^{\alpha-1}} G_{1}(t, s) .
\end{aligned}
$$

Therefore, $G_{1}(t, s) \geq \theta^{\alpha-1} G_{1}(\tau(s), s)$. Letting $\theta^{\alpha-1}=\gamma_{2}$, we have

$$
G_{1}(t, s) \geq \gamma_{2} G_{1}(\tau(s), s) .
$$


Case 3. If $s \in[0, \theta]$, from (i) of Proposition 2.1, it is clear that

$$
G_{1}(t, s)>0, \quad G_{1}(\tau(s), s)>0, \quad \forall t \in J_{\theta}, s \in(0, \theta] .
$$

In view of the Remarks 2.4-2.6, we have

$$
\begin{aligned}
& \lim _{s \rightarrow 0} \frac{G_{1}(t, s)}{G_{1}(\tau(s), s)} \\
& =\lim _{s \rightarrow 0} \frac{a t^{\alpha-1}(1-s)^{\alpha-1}+b(\alpha-1) t^{\alpha-1}(1-s)^{\alpha-2}-(a+b(\alpha-1))(t-s)^{\alpha-1}}{a(\tau(s))^{\alpha-1}(1-s)^{\alpha-1}+b(\alpha-1)(\tau(s))^{\alpha-1}(1-s)^{\alpha-2}-(a+b(\alpha-1))(\tau(s)-s)^{\alpha-1}} \\
& =\lim _{s \rightarrow 0}\left(\left(-a(\alpha-1) t^{\alpha-1}(1-s)^{\alpha-2}-b(\alpha-1) t^{\alpha-1}(1-s)^{\alpha-3}\right.\right. \\
& \left.\left.\quad+(\alpha-1)(a+b(\alpha-1))(t-s)^{\alpha-2}\right) /\left(\frac{d \bar{G}_{1}(\tau(s), s)}{d s}\right)\right) \\
& >0 .
\end{aligned}
$$

From (2.16), there exists a constant $\gamma_{3}$ such that

$$
G_{1}(t, s) \geq \gamma_{3} G_{1}(\tau(s), s)
$$

Letting $\gamma=\min \left\{\gamma_{1}, \gamma_{2}, \gamma_{3}\right\}$ and using (2.13), (2.15) and (2.17), it follows that (2.12) holds. This completes the proof.

Let

$$
\mu_{i}=\int_{0}^{1} h_{i}(t) t^{\alpha-1} d t
$$

Proposition 2.3 If $\mu_{i} \in[0, a-b(\alpha-1))$, then we have

(i) $G_{2 i}(t, s) \geq 0$ is continuous for all $t, s \in J, G_{2 i}(t, s)>0, \forall t, s \in(0,1)$;

(ii) $G_{2 i}(t, s) \leq \frac{1}{(a-b(\alpha-1))-\mu_{i}} \int_{0}^{1} h_{i}(t) G_{1}(t, s) d t, \forall t \in J, s \in(0,1)$.

Proof Using the properties of $G_{1}(t, s)$, definition of $G_{2 i}(t, s)$, it can easily be shown that (i) and (ii) hold.

Theorem 2.1 If $\mu_{i} \in[0, a-b(\alpha-1))$, the function $G_{i}(t, s)$ defined by (2.4) satisfies

(i) $G_{i}(t, s) \geq 0$ is continuous for all $t, s \in J, G_{i}(t, s)>0, \forall t, s \in(0,1)$;

(ii) $G_{i}(t, s) \leq G_{i}(s)$ for each $t, s \in J$, and

$$
\min _{t \in[\theta, 1-\theta]} G_{i}(t, s) \geq \gamma^{*} G_{i}(s), \quad \forall s \in J
$$

where

$$
\gamma^{*}=\min \left\{\gamma, \theta^{\alpha-1}\right\}, \quad G_{i}(s)=G_{1}(\tau(s), s)+G_{2 i}(1, s),
$$

$\tau(s)$ is defined by (2.9), $\gamma$ is defined in Proposition 2.2. 
Proof (i) From Proposition 2.1 and Proposition 2.3, we see that $G_{i}(t, s) \geq 0$ is continuous for all $t, s \in J$, and $G_{i}(t, s)>0, \forall t, s \in(0,1)$.

(ii) From (ii) of Proposition 2.1 and (ii) of Proposition 2.3, we have $G_{i}(t, s) \leq G_{i}(s)$ for each $t, s \in J$.

Now, we show that (2.19) holds.

In fact, from Proposition 2.2, we have

$$
\begin{aligned}
\min _{t \in J_{\theta}} G_{i}(t, s) & \geq \gamma G_{1}(\tau(s), s)+\frac{\theta^{\alpha-1}}{(a-b(\alpha-1))-\mu_{i}} \int_{0}^{1} h_{i}(t) G_{1}(t, s) d t \\
& \geq \gamma^{*}\left[G_{1}(\tau(s), s)+\frac{1}{(a-b(\alpha-1))-\mu_{i}} \int_{0}^{1} h_{i}(t) G_{1}(t, s) d t\right] \\
& =\gamma^{*} G_{i}(s), \quad \forall s \in J .
\end{aligned}
$$

Then the proof of Theorem 2.1 is completed.

Remark 2.8 From the definition of $\gamma^{*}$, it is clear that $0<\gamma^{*}<1$.

\section{Preliminaries}

Let $E=C[0,1], \mathbf{X}=\underbrace{E \times \cdots \times E}_{n}$, and, for any $\mathbf{x}=\left[x_{1}, x_{2}, \ldots, x_{n}\right]^{\top} \in \mathbf{X}$,

$$
\|\mathbf{X}\|=\sum_{i=1}^{n} \sup _{t \in J}\left|x_{i}(t)\right|
$$

Then $(\mathbf{X}, \cdot)$ is a real Banach space.

To establish the existence of positive solutions to system (1.1)-(1.2), for a fixed $\theta \in\left(t_{0}^{\prime}, \frac{1}{2}\right)$, we construct the cone $\mathbf{K}_{\theta}$ in $\mathbf{X}$ by

$$
\begin{aligned}
\mathbf{K}_{\theta}= & \left\{\mathbf{x}=\left(x_{1}, x_{2}, \ldots, x_{n}\right) \in \mathbf{X}: x_{i}(t) \geq 0, i=1,2, \ldots, n, t \in J,\right. \\
& \left.\min _{t \in[\theta, 1-\theta]} \sum_{i=1}^{n} x_{i}(t) \geq \gamma^{*}\|\mathbf{x}\|\right\},
\end{aligned}
$$

where $\gamma^{*}$ is defined in (2.20), and it is easy to see $\mathbf{K}_{\theta}$ is a closed convex cone of $\mathbf{X}$.

Let $\left\{\theta_{j}\right\}_{j=1}^{\infty}$ be such that $t_{j+1}^{\prime}<\theta_{j}<t_{j}^{\prime}, j=1,2, \ldots$. So we get $0<\cdots<t_{j+1}^{\prime}<\theta_{j}<t_{j}^{\prime}<\cdots<t_{3}^{\prime}<$ $\theta_{2}<t_{2}^{\prime}<\theta_{1}<t_{1}^{\prime}<\frac{1}{2}<1$. Then, for any $j \in \mathrm{N}$, we define the cone $\mathbf{K}_{\theta_{j}}$ by

$$
\mathbf{K}_{\theta_{j}}=\left\{\mathbf{x} \in \mathbf{X}: x_{i}(t) \geq 0, t \in J, i=1,2, \ldots, n, \min _{t \in\left[\theta_{j}, 1-\theta_{j}\right]} \sum_{i=1}^{n} x_{i}(t) \geq \gamma_{j}^{*}\|\mathbf{x}\|\right\},
$$

where

$$
\gamma_{j}^{*}=\min \left\{\gamma_{j}, \theta_{j}^{\alpha-1}\right\},
$$

here $\gamma_{j}=\min \left\{\gamma_{1 j}, \gamma_{2 j}, \gamma_{3 j}\right\}, \gamma_{1}, \gamma_{2}$ and $\gamma_{3}$ is similarly defined in (2.13), (2.14) and (2.17), respectively. It is easy to see $\mathbf{K}_{\theta_{j}}$ is a closed convex cone of $\mathbf{X}$. 
Also, for a positive number $\tau$, define $\mathbf{K}_{\tau \theta_{j}}$ by

$$
\mathbf{K}_{\tau \theta_{j}}=\left\{\mathbf{x} \in \mathbf{K}_{\theta_{j}}:\|\mathbf{x}\|<\tau\right\} .
$$

Let $\mathbf{T}_{\lambda}: \mathbf{K}_{\theta_{j}} \rightarrow \mathbf{X}$ be a map with components $\left(T_{\lambda}^{1}, T_{\lambda}^{2}, \ldots, T_{\lambda}^{i}, \ldots, T_{\lambda}^{n}\right)$. We understand that $\mathbf{T}_{\lambda} \mathbf{x}=\left(T_{\lambda}^{1} \mathbf{x}, T_{\lambda}^{2} \mathbf{x}, \ldots, T_{\lambda}^{i} \mathbf{x}, \ldots, T_{\lambda}^{n} \mathbf{x}\right)^{\top}$, where

$$
\left(T_{\lambda}^{i} \mathbf{x}\right)(t)=\lambda \int_{0}^{1} G_{i}(t, s) g_{i}(s) f_{i}(s, \mathbf{x}(s)) d s, \quad i=1,2, \ldots, n .
$$

Remark 3.1 It follows from Lemma 2.1 and the definition of $\mathbf{T}_{\lambda}$ that

$$
\mathbf{x}=\left[x_{1}, x_{2}, \ldots, x_{n}\right]^{\top} \in \mathbf{X}
$$

is a solution of system (1.1)-(1.2) if and only if $\mathbf{x}=\left[x_{1}, x_{2}, \ldots, x_{n}\right]^{\top}$ is a fixed point of operator $\mathbf{T}_{\lambda}$. And then $\mathbf{x}=\left[x_{1}, x_{2}, \ldots, x_{n}\right]^{\top} \in \mathbf{X}$ is a solution of the system (1.1)-(1.2) if and only if $x_{i}(i=1,2, \ldots, n)$ is a fixed point of operator $T_{\lambda}^{i}$.

Lemma 3.1 Assume that $\left(H_{1}\right)-\left(H_{4}\right)$ hold. Then $\mathbf{T}_{\lambda}\left(\mathbf{K}_{\theta_{j}}\right) \subset \mathbf{K}_{\theta_{j}}$ and $\mathbf{T}_{\lambda}: \mathbf{K}_{\theta_{j}} \rightarrow \mathbf{K}_{\theta_{j}}$ is a completely continuous.

Proof By the theory of matrix analysis, if we want to prove that $\mathbf{T}_{\lambda}\left(\mathbf{K}_{\theta_{j}}\right) \subset \mathbf{K}_{\theta_{j}}$ and $\mathbf{T}_{\lambda}$ : $\mathbf{K}_{\theta_{j}} \rightarrow \mathbf{K}_{\theta_{j}}$ is a completely continuous, then, for $i=1,2, \ldots, n$, we only prove that $T_{\lambda}^{i}\left(\mathbf{K}_{\theta_{j}}\right) \subset$ $\mathbf{K}_{\theta_{j}}$ and $T_{\lambda}^{i}: \mathbf{K}_{\theta_{j}} \rightarrow \mathbf{K}_{\theta_{j}}$ is a completely continuous.

Firstly, we prove that $T_{\lambda}^{i}\left(\mathbf{K}_{\theta_{j}}\right) \subset \mathbf{K}_{\theta_{j}}$. For $t \in J$, it follows from (ii) of Theorem 2.1 and (3.5) that

$$
\begin{aligned}
\left(T_{\lambda}^{i} \mathbf{x}\right)(t) & =\lambda \int_{0}^{1} G_{i}(t, s) g_{i}(s) f_{i}(s, \mathbf{x}(s)) d s \\
& \leq \lambda \int_{0}^{1} G_{i}(s) g_{i}(s) f_{i}(s, \mathbf{x}(s)) d s
\end{aligned}
$$

On the other hand, it follows from (2.19) and (3.5) that

$$
\begin{aligned}
\min _{t \in\left[\theta_{j}, 1-\theta_{j}\right]}\left(T_{\lambda}^{i} \mathbf{x}\right)(t) & =\min _{t \in\left[\theta_{j}, 1-\theta_{j}\right]} \lambda \int_{0}^{1} G_{i}(t, s) g_{i}(s) f_{i}(s, \mathbf{x}(s)) d s \\
& \geq \gamma_{j}^{*} \lambda \int_{0}^{1} G_{i}(s) g_{i}(s) f_{i}(s, \mathbf{x}(s)) d s \\
& \geq \gamma_{j}^{*}\left\|T_{\lambda}^{i} \mathbf{x}\right\| .
\end{aligned}
$$

This shows that $T_{\lambda}^{i}\left(\mathbf{K}_{\theta_{j}}\right) \subset \mathbf{K}_{\theta_{j}}$.

Next, by using similar arguments of Lemma 3.1 in [7] one can prove that the operator $T_{\lambda}^{i}: \mathbf{K}_{\theta_{j}} \rightarrow \mathbf{K}_{\theta_{j}}$ is completely continuous. So the proof of Lemma 3.1 is complete.

To obtain some of the norm inequalities in our main results, we employ the famous Hölder inequality. 
Lemma 3.2 (Hölder) Let $e \in L^{p}[a, b]$ with $p>1, h \in L^{q}[a, b]$ with $q>1$ and $\frac{1}{p}+\frac{1}{q}=1$. Then $e h \in L^{1}[a, b]$ and

$$
\|e h\|_{1} \leq\|e\|_{p}\|h\|_{q}
$$

Let $e \in L^{1}[a, b], h \in L^{\infty}[a, b]$. Then eh $\in L^{1}[a, b]$ and

$$
\|e h\|_{1} \leq\|e\|_{1}\|h\|_{\infty}
$$

Finally, we state the well-known fixed point theorems, which can be found in [56].

Lemma 3.3 Let $E$ be a Banach space, $K$ be a cone in $E$. Assume that $\Omega_{1}, \Omega_{2}$ are bounded open subsets in $E$ with $\theta \in \Omega_{1}$ and $\bar{\Omega}_{1} \subset \Omega_{2}$, where $\theta$ denotes the zero operator. Suppose $A: K \cap\left(\bar{\Omega}_{2} \backslash \Omega_{1}\right) \rightarrow K$ is completely continuous such that either

(i) $\|A x\| \leq\|x\|, \forall x \in K \cap \partial \Omega_{1} ;\|A x\| \geq\|x\|, \forall x \in K \cap \partial \Omega_{2}$;

(ii) $\|A x\| \leq\|x\|, \forall x \in K \cap \partial \Omega_{2} ;\|A x\| \geq\|x\|, \forall x \in K \cap \partial \Omega_{1}$.

Then $A$ has a fixed point in $K \cap\left(\bar{\Omega}_{2} \backslash \Omega_{1}\right)$.

Lemma 3.4 Let $E$ be a real Banach space and let $K$ be a cone in $E$. For $r>0$, we define $K_{r}=\{x \in K:\|x\|<r\}$. Assume that $T: \bar{K}_{r} \rightarrow K$ is completely continuous such that $T x \neq x$ for $x \in \partial K r=\{x \in K:\|x\|=r\}$.

(i) If $\|T x\| \geq\|x\|$ for $x \in \partial K_{r}$, then $\mathbf{i}\left(T, K_{r}, K\right)=0$.

(ii) If $\|T x\| \leq\|x\|$ for $x \in \partial K_{r}$, then $\mathbf{i}\left(T, K_{r}, K\right)=1$.

Remark 3.2 It is well known that Lemma 3.3 and Lemma 3.4 have been instrumental in proving the existence of positive solutions to various boundary value problems for integerorder or fractional-order differential equations-for details; see Sect. 6.

\section{The existence of denumerably many positive solutions}

In this section, we establish the existence of the denumerably many positive solutions for system (1.1)-(1.2). We give our main results in the cases with $g_{i} \in L^{P}[0,1] ; p>1, p=1$ and $p=\infty$.

Firstly, we consider the case $p>1$.

Theorem 4.1 Assume that $\left(H_{1}\right)-\left(H_{4}\right)$ hold. Let $\left\{r_{j}\right\}_{j=1}^{\infty}$ and $\left\{R_{j}\right\}_{j=1}^{\infty}$ be such that

$$
R_{j+1}<\gamma_{j}^{*} r_{j}<r_{j}<R_{j}, \quad j=1,2, \ldots
$$

For each natural number $j$, we assume that $\mathbf{f}$ satisfies

$\left(H_{5}\right)$ For any $t \in J,\|\mathbf{x}\| \in\left[0, R_{j}\right], f_{i}(t, \mathbf{x}) \leq L R_{j}$, where

$$
\begin{aligned}
0<L & \leq \max \left\{\frac{1}{n \lambda\left\|G_{i}\right\|_{q}\left\|g_{i}\right\|_{p}}, \frac{1}{n \lambda\left\|G_{i}\right\|_{1}\left\|g_{i}\right\|_{\infty}}, \frac{1}{n \lambda M_{i}\left\|g_{i}\right\|_{1}}\right\} \\
M_{i} & =\max _{s \in J} G_{i}(s)
\end{aligned}
$$

$\left(H_{6}\right)$ for any $t \in\left[\theta_{j}, 1-\theta_{j}\right],\|\mathbf{x}\| \in\left[\gamma_{j}^{*} r_{j}, r_{j}\right], f_{i}(t, \mathbf{x}) \geq l r_{j}$, where $l>0$. 
Then there exists $\lambda_{0}>0$, such that, for $\lambda>\lambda_{0}$, system (1.1)-(1.2) has denumerably many positive solutions $\left\{\mathbf{x}_{j}\right\}_{j=1}^{\infty}$ such that

$$
r_{j} \leq\left\|\mathbf{x}_{j}\right\| \leq R_{j}, \quad j=1,2, \ldots
$$

Proof Let $\lambda_{0}=\sup \left\{\lambda_{j}\right\}, \lambda_{j}=\frac{1}{\gamma_{j}^{*} N_{i} l \int_{\theta_{j}}^{1-\theta_{j}} G_{i}(s) d s}, i=1,2, \ldots, n, j=1,2, \ldots$ Then, for any $\lambda>\lambda_{0}$, (3.5) and Lemma 3.1 imply that $\mathbf{T}_{\lambda}$ and $T_{\lambda}^{i}(i=1,2, \ldots, n)$ are all completely continuous.

We consider the open subset sequences $\left\{\Omega_{1, j}\right\}_{j=1}^{\infty}$ and $\left\{\Omega_{2, j}\right\}_{j=1}^{\infty}$ of $\mathbf{X}$

$$
\begin{gathered}
\left\{\Omega_{1, j}\right\}_{j=1}^{\infty}=\left\{\mathbf{x} \in \mathbf{X}:\|\mathbf{x}\|<R_{j}\right\} ; \\
\left\{\Omega_{2, j}\right\}_{j=1}^{\infty}=\left\{\mathbf{x} \in \mathbf{X}:\|\mathbf{x}\|<r_{j}\right\} .
\end{gathered}
$$

Let $\left\{\theta_{j}\right\}_{j=1}^{\infty}$ be as in Sect. 3 and note that $0<t_{j+1}^{\prime}<\theta_{j}<t_{j}^{\prime}<\frac{1}{2}, j=1,2, \ldots$

For fixed $j$, we assume that $\mathbf{x} \in K_{\theta_{j}} \cap \partial \Omega_{2, j}$, then for any $t \in J$

$$
r_{j}=\|\mathbf{x}\|=\sum_{i=1}^{n} \sup _{t \in J}\left|x_{i}(t)\right| \geq \min _{t \in\left[\theta_{j}, 1-\theta_{j}\right]} \sum_{i=1}^{n} x_{i}(t) \geq \gamma_{j}^{*}\|\mathbf{x}\|=\gamma_{j}^{*} r_{j}
$$

Noticing (2.19) and (3.5), for all $\mathbf{x} \in K_{\theta_{j}} \cap \partial \Omega_{2, j}$, by $\left(H_{1}\right)$ and $\left(H_{6}\right)$, we have

$$
\begin{aligned}
\left\|\mathbf{T}_{\lambda} \mathbf{x}\right\| & \geq \sup _{t \in J}\left|\left(T_{\lambda}^{i} \mathbf{x}\right)(t)\right| \\
& =\lambda \sup _{t \in J} \int_{0}^{1} G_{i}(t, s) g_{i}(s) f_{i}(s, \mathbf{x}(s)) d s \\
& \geq \min _{t \in\left[\theta_{j}, 1-\theta_{j}\right]} \lambda \int_{0}^{1} G_{i}(t, s) g_{i}(s) f_{i}(s, \mathbf{x}(s)) d s \\
& \geq \gamma_{j}^{*} \lambda N_{i} \int_{0}^{1} G_{i}(s) f_{i}(s, \mathbf{x}(s)) d s \\
& \geq \gamma_{j}^{*} \lambda N_{i} \int_{\theta_{j}}^{1-\theta_{j}} G_{i}(s) f_{i}(s, \mathbf{x}(s)) d s \\
& \geq \gamma_{j}^{*} \lambda N_{i} l \int_{\theta_{j}}^{1-\theta_{j}} G_{i}(s) d s r_{j} \\
& >\lambda_{0} \gamma_{j}^{*} N_{i} l \int_{\theta_{j}}^{1-\theta_{j}} G_{i}(s) d s r_{j} \\
& =r_{j}=\|\mathbf{x}\|,
\end{aligned}
$$

which shows that

$$
\left\|T_{\lambda} \mathbf{x}\right\| \geq\|\mathbf{x}\|, \quad \forall \mathbf{x} \in K_{\theta_{j}} \cap \partial \Omega_{2, j} .
$$

On the other hand, for all $t \in J, \mathbf{x} \in K_{\theta_{j}} \cap \partial \Omega_{1, j}$, then $\|\mathbf{x}\|=R_{j}$. 
Noticing Theorem 2.1 and (3.5), for all $t \in J, \mathbf{x} \in K_{\theta_{j}} \cap \partial \Omega_{1, j}$, by $\left(H_{5}\right)$, we have

$$
\begin{aligned}
\left(T_{\lambda}^{i} \mathbf{x}\right)(t) & =\lambda \int_{0}^{1} G_{i}(t, s) g_{i}(s) f_{i}(s, \mathbf{x}(s)) d s \\
& \leq \lambda \int_{0}^{1} G_{i}(t, s) g_{i}(s) f_{i}(s, \mathbf{x}(s)) d s \\
& \leq \lambda \int_{0}^{1} G_{i}(s) g_{i}(s) f_{i}(s, \mathbf{x}(s)) d s \\
& \leq \lambda \int_{0}^{1}\left\|G_{i}\right\|_{q}\left\|g_{i}\right\|_{p} f_{i}(s, \mathbf{x}(s)) d s \\
& \leq \lambda\left\|G_{i}\right\|_{q}\left\|g_{i}\right\|_{p} \int_{0}^{1} f_{i}(s, \mathbf{x}(s)) d s \\
& \leq \lambda\left\|G_{i}\right\|_{q}\left\|g_{i}\right\|_{p} L R_{j} \\
& \leq \frac{1}{n} R_{j}=\frac{1}{n}\|\mathbf{x}\|,
\end{aligned}
$$

which shows that

$$
\left\|\mathbf{T}_{\lambda} \mathbf{x}\right\|=\sum_{i=1}^{n} \sup _{t \in J}\left|\left(T_{\lambda}^{i} \mathbf{x}\right)(t)\right| \leq\|\mathbf{x}\|, \quad \forall \mathbf{x} \in K_{\theta_{j}} \cap \partial \Omega_{1, j}
$$

Applying Lemma 3.3 to (4.2) and (4.3) yields that operator $\mathbf{T}_{\lambda}$ has a fixed point $\mathbf{x}_{j} \in$ $K_{\theta_{j}} \cap\left(\bar{\Omega}_{2, j} / \Omega_{1, j}\right)$ such that $r_{j} \leq\left\|\mathbf{x}_{j}\right\| \leq R_{j}$. And then it follows from Remark 3.2 that system (1.1)-(1.2) has a solution $\mathbf{x}_{j}=\left(x_{1 j}, x_{2 j}, \ldots, x_{n j}\right)^{\top}$. Since $j \in \mathcal{N}$ was arbitrary, the proof is complete.

The following theorem deals with the case $p=\infty$.

Theorem 4.2 Assume that $\left(H_{1}\right)-\left(H_{4}\right)$ hold. Let $\left\{r_{j}\right\}_{j=1}^{\infty}$ and $\left\{R_{j}\right\}_{j=1}^{\infty}$ be such that

$$
R_{j+1}<\gamma_{j}^{*} r_{j}<r_{j}<R_{j}, \quad j=1,2, \ldots
$$

For each natural number $j$, we assume that $\mathbf{f}$ satisfies $\left(H_{5}\right)$ and $\left(H_{6}\right)$, then there exists $\lambda_{0}>0$, such that, for $\lambda>\lambda_{0}$, system (1.1)-(1.2) has denumerably many positive solutions $\left\{\mathbf{x}_{j}(t)\right\}_{j=1}^{\infty}$ such that

$$
r_{j} \leq\left\|\mathbf{x}_{j}\right\| \leq R_{j}, \quad j=1,2, \ldots
$$

Proof Let $\left\|G_{i}\right\|_{1}\left\|g_{i}\right\|_{\infty}$ replace $\left\|G_{i}\right\|_{q}\left\|g_{i}\right\|_{p}$ and repeat the previous argument.

Finally, we consider the case of $p=1$.

Theorem 4.3 Assume that $\left(H_{1}\right)-\left(H_{4}\right)$ hold. Let $\left\{r_{j}\right\}_{j=1}^{\infty}$ and $\left\{R_{j}\right\}_{j=1}^{\infty}$ be such that

$$
R_{j+1}<\gamma_{j}^{*} r_{j}<r_{j}<R_{j}, \quad j=1,2, \ldots
$$


For each natural number $j$, we assume that $\mathbf{f}$ satisfies $\left(H_{5}\right)$ and $\left(H_{6}\right)$, then there exists $\lambda_{0}>0$, such that, for $\lambda>\lambda_{0}$, system (1.1)-(1.2) has denumerably many positive solutions $\left\{\mathbf{x}_{j}(t)\right\}_{j=1}^{\infty}$ such that

$$
r_{j} \leq\left\|\mathbf{x}_{j}\right\| \leq R_{j}, \quad j=1,2, \ldots
$$

Proof Let $M_{i}\left\|g_{i}\right\|_{1}$ replace $\left\|G_{i}\right\|_{q}\left\|g_{i}\right\|_{p}$ and repeat the previous argument.

Corollary 4.1 Assume that $\left(H_{1}\right)-\left(H_{4}\right)$ hold. Let $\left\{r_{j}\right\}_{j=1}^{\infty}$ and $\left\{R_{j}\right\}_{j=1}^{\infty}$ be such that

$$
R_{j+1}<\gamma_{j}^{*} r_{j}<r_{j}<R_{j}, \quad j=1,2, \ldots
$$

For each natural number $j$, we assume that $\mathbf{f}$ satisfies:

$\left(H_{5}^{\prime}\right)$ for any $t \in J,\|\mathbf{x}\| \in\left[0, r_{j}\right], f(t, \mathbf{x}) \leq L r_{j}$, where $L$ is defined in (4.1);

$\left(H_{6}^{\prime}\right)$ for any $t \in\left[\theta_{j}, 1-\theta_{j}\right],\|\mathbf{x}\| \in\left[\gamma_{j}^{*} R_{j}, R_{j}\right], f(t, \mathbf{x}) \geq l R_{j}$, where $l>0$.

Then there exists $\lambda_{0}>0$, such that, for $\lambda>\lambda_{0}$, system (1.1)-(1.2) has denumerably many positive solutions $\left\{\mathbf{x}_{j}(t)\right\}_{j=1}^{\infty}$ such that

$$
r_{j} \leq\left\|\mathbf{x}_{j}\right\| \leq R_{j}, \quad j=1,2, \ldots
$$

\section{The existence of two infinite families of positive solutions}

In this section, we use Lemma 3.4 to establish the existence of two infinite families of positive solutions for system (1.1)-(1.2).

For ease of expression, we introduce the following notations:

$$
\begin{aligned}
& \left(f_{0}^{\tau}\right)^{i}=\max \left\{\max _{t \in J} \frac{f_{i}(t, \mathbf{x})}{\tau}, 0 \leq\|\mathbf{x}\| \leq \tau\right\}, \quad F_{0}^{\tau}=\max _{1 \leq i \leq n}\left(f_{0}^{\tau}\right)^{i}, \\
& \left(f_{\gamma_{j}^{*} \tau}^{\tau}\right)^{i}=\min \left\{\min _{t \in\left[\theta_{j}, 1-\theta_{j}\right]} \frac{f_{i}(t, \mathbf{x})}{\tau}, \gamma_{j}^{*} \tau \leq\|\mathbf{x}\| \leq \tau\right\}, \quad F_{\gamma_{j}^{*} \tau}^{\tau}=\min _{1 \leq i \leq n}\left(f_{\gamma_{j}^{*} \tau}^{\tau}\right)^{i},
\end{aligned}
$$

where $i=1,2, \ldots, n, j=1,2, \ldots$.

In this section, we also consider the following three cases for $g_{i} \in L^{P}[0,1]: p>1, p=1$ and $p=\infty$. Case $p>1$ is treated in the following theorem.

Theorem 5.1 Assume that $\left(H_{1}\right)-\left(H_{4}\right)$ hold. Let $\left\{r_{j}\right\}_{j=1}^{\infty},\left\{\eta_{j}\right\}_{j=1}^{\infty}$ and $\left\{R_{j}\right\}_{j=1}^{\infty}$ be such that

$$
R_{j+1}<\sigma_{j} r_{j}<r_{j}<\sigma_{j} \eta_{j}<\eta_{j}<R_{j}, \quad j=1,2, \ldots
$$

Furthermore, for each natural number $j$, we assume that $\mathbf{f}$ satisfies

$\left(H_{7}\right) F_{0}^{r_{j}} \leq L$ and $F_{0}^{R_{j}} \leq L$, where $L$ is defined in (4.1);

$\left(H_{8}\right) F_{\gamma_{j}^{*} \eta_{j}}^{\eta_{j}} \geq l$, where $l>0$.

Then there exists $\lambda_{0}>0$ such that, for $\lambda>\lambda_{0}$, system (1.1)-(1.2) has two infinite families of positive solutions $\left\{\mathbf{x}_{j}^{(1)}(t)\right\}_{j=1}^{\infty},\left\{\mathbf{x}_{j}^{(2)}(t)\right\}_{j=1}^{\infty}$ and $\left\|\mathbf{x}_{j}^{(1)}\right\|>\gamma_{j}^{*} \eta_{j}$.

Proof Let $\lambda_{0}$ be defined as in Theorem 4.1. Then, for any $\lambda>\lambda_{0}$, (3.5) and Lemma 3.1 imply that $\mathbf{T}_{\lambda}$ and $T_{\lambda}^{i}(i=1,2, \ldots, n)$ are all completely continuous.

Let $t \in J, \mathbf{x} \in \partial \mathbf{K}_{r_{j} \theta_{j}}$. Then $\|\mathbf{x}\|=r_{j}$. 
Therefore, for any $\mathbf{x} \in \partial \mathbf{K}_{r_{j} \theta_{j}}$, it follows from $\left(H_{7}\right)$ that

$$
\begin{aligned}
\left(T_{\lambda}^{i} \mathbf{x}\right)(t) & =\lambda \int_{0}^{1} G_{i}(t, s) g_{i}(s) f_{i}(s, \mathbf{x}(s)) d s \\
& \leq \lambda \int_{0}^{1} G_{i}(t, s) g_{i}(s) f_{i}(s, \mathbf{x}(s)) d s \\
& \leq \lambda \int_{0}^{1} G_{i}(s) g_{i}(s) f_{i}(s, \mathbf{x}(s)) d s \\
& \leq \lambda \int_{0}^{1}\left\|G_{i}\right\|_{q}\left\|g_{i}\right\|_{p} f_{i}(s, \mathbf{x}(s)) d s \\
& \leq \lambda\left\|G_{i}\right\|_{q}\left\|g_{i}\right\|_{p} \int_{0}^{1} f_{i}(s, \mathbf{x}(s)) d s \\
& \leq \lambda\left\|G_{i}\right\|_{q}\left\|g_{i}\right\|_{p} L r_{j} \\
& \leq \frac{1}{n} r_{j}=\frac{1}{n}\|\mathbf{x}\|
\end{aligned}
$$

which shows that

$$
\left\|\mathbf{T}_{\lambda} \mathbf{x}\right\|=\sum_{i=1}^{n} \sup _{t \in J}\left|\left(T_{\lambda}^{i} \mathbf{x}\right)(t)\right| \leq\|\mathbf{x}\|, \quad \forall \mathbf{x} \in \partial \mathbf{K}_{r_{j}} \theta_{j}
$$

And then, by Lemma 3.4, we get

$$
\mathbf{i}\left(\mathbf{T}_{\lambda}, \mathbf{K}_{r_{j} \theta_{i}}, \mathbf{K}_{\theta_{j}}\right)=1
$$

Similarly, for $\mathbf{x} \in \partial \mathbf{K}_{R_{j} \theta_{j}}$, we have $\left\|\mathbf{T}_{\lambda} \mathbf{x}\right\| \leq\|\mathbf{x}\|$, and it follows from Lemma 3.4 that

$$
\mathbf{i}\left(\mathbf{T}_{\lambda}, \mathbf{K}_{R_{j} \theta_{j}}, \mathbf{K}_{\theta_{j}}\right)=1
$$

On the other hand, letting

$$
\mathbf{x} \in \overline{\mathbf{K}}_{\gamma_{j}^{*} \eta_{j} \theta_{j}}^{\eta_{j}}=\left\{\mathbf{x} \in \mathbf{K}_{\theta_{j}}:\|\mathbf{x}\| \leq \eta_{j}, \min _{t \in\left[\theta_{j}, 1-\theta_{j}\right]} \sum_{i=1}^{n} x_{i}(t) \geq \gamma_{j}^{*} \eta_{j}\right\},
$$

then $\|\mathbf{x}\| \leq \eta_{j}$. And hence, similar to the proof of (5.2), we have

$$
\left\|\mathbf{T}_{\lambda} \mathbf{x}\right\| \leq \eta_{j}
$$

Furthermore, for $\mathbf{x} \in \overline{\mathbf{K}}_{\gamma_{j}^{*} \eta_{j} \theta_{j}}^{\eta_{j}}$, we have

$$
\|\mathbf{x}\| \leq \eta_{j}, \quad t \in J, \min _{t \in\left[\theta_{j}, 1-\theta_{j}\right]} \sum_{i=1}^{n} x_{i}(t) \geq \gamma_{j}^{*} \eta_{j}
$$


and then it follows from $\left(H_{8}\right)$ that

$$
\begin{aligned}
\left(\mathbf{T}_{\lambda} \mathbf{x}\right)(t) & \geq \sup _{t \in J}\left|\left(T_{\lambda}^{i} \mathbf{x}\right)(t)\right| \\
& \geq \lambda \int_{0}^{1} G_{i}(t, s) g_{i}(s) f_{i}(s, \mathbf{x}(s)) d s \\
& \geq \min _{t \in\left[\theta_{j}, 1-\theta_{j}\right]} \lambda \int_{0}^{1} G_{i}(t, s) g_{i}(s) f_{i}(s, \mathbf{x}(s)) d s \\
& \geq \gamma_{j}^{*} \lambda N_{i} \int_{0}^{1} G_{i}(s) f_{i}(s, \mathbf{x}(s)) d s \\
& \geq \gamma_{j}^{*} \lambda N_{i} \int_{\theta_{j}}^{1-\theta_{j}} G_{i}(s) f_{i}(s, \mathbf{x}(s)) d s \\
& \geq \gamma_{j}^{*} \eta_{j} \lambda N_{i} l \int_{\theta_{j}}^{1-\theta_{j}} G_{i}(s) d s \\
& >\lambda_{0} \gamma_{j}^{*} \eta_{j} N_{i} l \int_{\theta_{j}}^{1-\theta_{j}} G_{i}(s) d s \\
& =\eta_{j}=\|\mathbf{x}\| .
\end{aligned}
$$

Letting $\mathbf{x}_{0}=\left(x_{0}^{1}, x_{0}^{2}, \ldots, x_{0}^{i}, \ldots, x_{0}^{n}\right)$ and $\mathbf{F}(t, \mathbf{x})=(1-t) \mathbf{T}_{\lambda} \mathbf{x}+t \mathbf{x}_{0}$, where $x_{0}^{i} \equiv \frac{\gamma_{j}^{*} \eta_{j}+\eta_{j}}{2}, i=$ $1,2, \ldots, n$, then $\mathbf{F}: J \times \overline{\mathbf{K}}_{\gamma_{j}^{*} \eta_{j} \theta_{j}}^{\eta_{j}} \rightarrow \mathbf{K}_{\theta_{j}}$ is completely continuous, and from the analysis above, we obtain, for $(t, \mathbf{x}) \in J \times \overline{\mathbf{K}}_{\gamma_{j}^{*} \eta_{j} \theta_{j}}^{\eta_{i}}$

$$
\mathbf{F}(t, \mathbf{x}) \in \overline{\mathbf{K}}_{\gamma_{j}^{*} \eta_{j} \theta_{j}}^{\eta_{j}}
$$

Therefore, for $t \in J, \mathbf{x} \in \overline{\mathbf{K}}_{\sigma_{j} \eta_{j} \theta_{j}}^{\eta_{j}}$, we have $\mathbf{F}(t, \mathbf{x}) \neq \mathbf{x}$. Hence, by the normality property and the homotopy invariance property of the fixed point index, we obtain

$$
\mathbf{i}\left(\mathbf{T}_{\lambda}, \mathbf{K}_{\gamma_{j}^{*} \eta_{j} \theta_{j}}^{\eta_{j}}, \mathbf{K}_{\theta_{j}}\right)=\mathbf{i}\left(\mathbf{x}_{0}, \mathbf{K}_{\gamma_{j}^{*} \eta_{j} \theta_{j}}^{\eta_{j}}, \mathbf{K}_{\theta_{j}}\right)=1
$$

Consequently, by the solution property of the fixed point index, $\mathbf{T}_{\lambda}$ has a fixed point $\mathbf{x}_{j}^{(1)}$ and $\mathbf{x}_{j}^{(1)} \in \overline{\mathbf{K}}_{\gamma_{j}^{*} \eta_{j} \theta_{j}}^{\eta_{j}}$. By Remark 3.1, it follows that $\mathbf{x}_{j}^{(1)}$ is a solution to system (1.1)-(1.2), and

$$
\left.\left\|\mathbf{x}_{j}^{(1)}\right\| \geq \min _{t \in\left[\theta_{j}, 1-\theta_{j}\right]} \sum_{i=1}^{n} x_{j}^{(1 i)}\right)(t)>\gamma_{j}^{*} \eta_{j}
$$

On the other hand, from (5.3), (5.4) and (5.8) together with the additivity of the fixed point index, we get

$$
\begin{aligned}
& \mathbf{i}\left(\mathbf{T}_{\lambda}, \mathbf{K}_{R_{j} \theta_{j}} /\left(\overline{\mathbf{K}}_{r_{j} \theta_{j}} \cup \overline{\mathbf{K}}_{\gamma_{j}^{*} \eta_{j} \theta_{j}}^{\eta_{j}}\right), \mathbf{K}_{\theta_{j}}\right) \\
& \quad=\mathbf{i}\left(\mathbf{T}_{\lambda}, \mathbf{K}_{R_{j} \theta_{j}}, \mathbf{K}_{\theta_{j}}\right)-\mathbf{i}\left(\mathbf{T}_{\lambda}, \overline{\mathbf{K}}_{\gamma_{j}^{*} \eta_{j} \theta_{j}}^{\eta_{j}}, \mathbf{K}_{\theta_{j}}\right)-\mathbf{i}\left(\mathbf{T}_{\lambda}, \overline{\mathbf{K}}_{r_{j} \theta_{j}}, \mathbf{K}_{\theta_{j}}\right) \\
& \quad=1-1-1=-1 .
\end{aligned}
$$


Hence, by the solution property of the fixed point index, $\mathbf{T}_{\lambda}$ has a fixed point $\mathbf{x}_{j}^{(2)}$ and $\mathbf{x}_{j}^{(2)} \in \mathbf{K}_{R_{j}} /\left(\overline{\mathbf{K}}_{r_{j}} \cup \overline{\mathbf{K}}_{\gamma_{j}^{*} \eta_{j} \theta_{j}}^{\eta_{j}}\right)$. Since $j \in \mathcal{N}$ was arbitrary, the proof is complete.

The following corollary deals with the case $p=\infty$.

Corollary 5.1 Assume that $\left(H_{1}\right)-\left(H_{4}\right)$ hold. Let $\left\{r_{j}\right\}_{j=1}^{\infty},\left\{\eta_{j}\right\}_{j=1}^{\infty}$ and $\left\{R_{j}\right\}_{j=1}^{\infty}$ satisfy (5.1). Furthermore, for each natural number $j$, we assume that $\mathbf{f}$ satisfies $\left(H_{7}\right)$ and $\left(H_{8}\right)$. Then there exist $\lambda_{0}>0$ such that, for $\lambda>\lambda_{0}$, system (1.1)-(1.2) has two infinite families of positive solutions $\left\{\mathbf{x}_{j}^{(1)}(t)\right\}_{j=1}^{\infty},\left\{\mathbf{x}_{j}^{(2)}(t)\right\}_{j=1}^{\infty}$ and $\left\|\mathbf{x}_{j}^{(1)}\right\|>\gamma_{j}^{*} \eta_{j}$.

Proof Let $\left\|G_{i}\right\|_{1}\left\|g_{i}\right\|_{\infty}$ replace $\left\|G_{i}\right\|_{q}\left\|g_{i}\right\|_{p}$ and repeat the argument above.

Finally, we consider the case of $p=1$.

Corollary 5.2 Assume that $\left(H_{1}\right)-\left(H_{4}\right)$ hold. Let $\left\{r_{j}\right\}_{j=1}^{\infty},\left\{\eta_{j}\right\}_{j=1}^{\infty}$ and $\left\{R_{j}\right\}_{j=1}^{\infty}$ satisfy (5.1). Furthermore, for each natural number $j$, we assume that $\mathbf{f}$ satisfies $\left(H_{7}\right)$ and $\left(H_{8}\right)$. Then there exist $\lambda_{0}>0$ such that, for $\lambda>\lambda_{0}$, system (1.1)-(1.2) has two infinite families of positive solutions $\left\{\mathbf{x}_{j}^{(1)}(t)\right\}_{j=1}^{\infty},\left\{\mathbf{x}_{j}^{(2)}(t)\right\}_{j=1}^{\infty}$ and $\left\|\mathbf{x}_{j}^{(1)}\right\|>\gamma_{j}^{*} \eta_{j}$.

Proof Let $M_{i}\left\|g_{i}\right\|_{1}$ replace $\left\|G_{i}\right\|_{q}\left\|g_{i}\right\|_{p}$ and repeat the previous argument. Similar to the proof of Theorem 5.1, we can get Corollary 5.2.

Corollary 5.3 Assume that $\left(H_{1}\right)-\left(H_{4}\right)$ hold. Let $\left\{r_{j}\right\}_{j=1}^{\infty},\left\{\eta_{j}\right\}_{j=1}^{\infty}$ and $\left\{R_{j}\right\}_{j=1}^{\infty}$ satisfy (5.1). Furthermore, for each natural number $j$, we assume that $\mathbf{f}$ satisfies $\left(H_{8}\right)$. Then there exist $\lambda_{0}>0$ such that, for $\lambda>\lambda_{0}$, system (1.1)-(1.2) has one infinite family of positive solutions.

If we replace $\left(H_{7}\right)$ by the following condition:

$\left(H_{7}^{\prime}\right) F_{0}^{r_{j}} \leq L$ or $F_{0}^{R_{j}} \leq L$, where $L$ is defined in (4.1), then we have the following corollary.

Corollary 5.4 Assume that $\left(H_{1}\right)-\left(H_{4}\right)$ hold. Let $\left\{r_{j}\right\}_{j=1}^{\infty},\left\{\eta_{j}\right\}_{j=1}^{\infty}$ and $\left\{R_{j}\right\}_{j=1}^{\infty}$ satisfy (5.1). Furthermore, for each natural number $j$, we assume that $\mathbf{f}$ satisfies $\left(H_{7}^{\prime}\right)$. Then for all $\lambda>0$, system (1.1)-(1.2) has one infinite family of nonnegative solutions.

\section{Comments and remarks}

In this section, we give some comments and remarks associated with system (1.1)-(1.2).

It is well known that Lemma 3.3 and Lemma 3.4 have been instrumental in proving the existence of positive solutions to various boundary value problems for integer-order differential equations. See for instance $[44-46,51]$ and the references therein. Several authors have investigated boundary value problems of fractional differential equations; see for instance $[8,11,13]$.

However, it is not difficult to see that if $\left(H_{1}\right)-\left(H_{4}\right)$ hold, and $\mathbf{f}$ satisfies $\left(H_{6}\right)$, by using Lemma 3.3, we can not obtain the results of Corollary 5.3.

At the same time, it is not difficult to see that $\left(H_{2}\right)$ plays an important role in the proof that system (1.1)-(1.2) has two infinite families of positive solutions. However, if the condition $\left(\mathrm{H}_{2}\right)$ does not hold, then we can only obtain the existence results of one and two positive solution for system (1.1)-(1.2). 
Theorem 6.1 Assume that $\left(H_{1}\right),\left(H_{3}\right)$ and $\left(H_{4}\right)$ hold. Furthermore, we assume that $\mathbf{f}$ satisfies:

$\left(H_{5}^{\prime}\right)$ for any $t \in J,\|\mathbf{x}\| \in[0, R], f_{i}(t, \mathbf{x}) \leq L R$, where $L$ satisfies (4.1);

$\left(H_{6}^{\prime}\right)$ for any $t \in[\theta, 1-\theta],\|\mathbf{x}\| \in\left[\gamma^{*} r, r\right], f_{i}(t, \mathbf{x}) \geq$ lr, where $l>0$.

Letting $0<r<R$, then there exists $\lambda_{0}>0$, such that, for $\lambda>\lambda_{0}$, system (1.1)-(1.2) has at least one positive solution $\mathbf{x}$ with

$$
r \leq\|\mathbf{x}\| \leq R
$$

Theorem 6.2 Assume that $\left(H_{1}\right),\left(H_{3}\right),\left(H_{4}\right)$ hold and $0<r<\eta<R$. Furthermore, we assume that $\mathbf{f}$ satisfies

$\left(H_{7}^{\prime \prime}\right) F_{0}^{r} \leq L$ and $F_{0}^{R} \leq L$, where $L$ is defined in (4.1);

$\left(H_{8}^{\prime}\right) F_{\sigma \eta}^{\eta} \geq l$, where $l>0$.

Then there exist $\lambda_{0}>0$ such that, for $\lambda>\lambda_{0}$, system (1.1)-(1.2) has at least two positive solutions $\mathbf{x}^{(1)}, \mathbf{x}^{(2)}$ and $\sum_{i=1}^{n} x_{i}^{(1)}(t)>\gamma^{*} \eta, \forall t \in[\theta, 1-\theta]$.

Remark 6.1 If $\left(H_{2}\right)$ does not hold, we can also consider the other cases.

To this aim, we begin by introducing the notations

$$
\begin{aligned}
& f_{i}^{0}=\limsup _{\|\mathbf{y}\| \rightarrow 0} \max _{t \in J} \frac{f_{i}(t, \mathbf{y})}{\|\mathbf{y}\|}, \quad f_{i}^{\infty}=\limsup _{\|\mathbf{y}\| \rightarrow \infty} \max _{t \in J} \frac{f_{i}(t, \mathbf{y})}{\|\mathbf{y}\|}, \\
& \mathbf{K}_{r, R}=\left\{\mathbf{x} \mid \mathbf{x} \in \mathbf{K}_{\theta}, r<\|\mathbf{x}\|<R\right\} .
\end{aligned}
$$

Theorem 6.3 Assume that $\left(H_{1}\right),\left(H_{3}\right)$ and $\left(H_{4}\right)$ hold. Furthermore, let the following two conditions hold:

(i) $f_{i}^{0}=0$ or $f_{i}^{\infty}=0$;

(ii) There exist $\rho>0, \delta>0$, such that $f_{i}(t, \mathbf{x}) \geq \delta$ for $\|\mathbf{x}\| \geq \rho, t \in J$

be satisfied, then there exists $\lambda_{0}>0$ such that, for all $\lambda>\lambda_{0}$, system (1.1)-(1.2) has at least one positive solution $\mathbf{x}^{*}$.

Proof Considering $f_{i}^{0}=0$, there exists $0<r<\rho$ such that $f_{i}(t, \mathbf{x}) \leq \varepsilon_{1} r$, for $0 \leq\|\mathbf{x}\| \leq r$, $t \in J$, where $\varepsilon_{1}>0$ satisfies $\lambda \varepsilon_{1}\left\|G_{i}\right\|_{q}\left\|g_{i}\right\|_{p} \leq \frac{1}{n}$.

So, for $x \in \partial K_{r \theta}$, we have from (3.5)

$$
\begin{aligned}
\left(T_{\lambda}^{i} \mathbf{x}\right)(t) & =\lambda \int_{0}^{1} G_{i}(t, s) g_{i}(s) f_{i}(s, \mathbf{x}(s)) d s \\
& \leq \lambda \int_{0}^{1} G_{i}(t, s) g_{i}(s) f_{i}(s, \mathbf{x}(s)) d s \\
& \leq \lambda \int_{0}^{1} G_{i}(s) g_{i}(s) f_{i}(s, \mathbf{x}(s)) d s \\
& \leq \lambda \int_{0}^{1}\left\|G_{i}\right\|_{q}\left\|g_{i}\right\|_{p} f_{i}(s, \mathbf{x}(s)) d s \\
& \leq \lambda\left\|G_{i}\right\|_{q}\left\|g_{i}\right\|_{p} \int_{0}^{1} f_{i}(s, \mathbf{x}(s)) d s
\end{aligned}
$$




$$
\begin{aligned}
& \leq \lambda\left\|G_{i}\right\|_{q}\left\|g_{i}\right\|_{p} \varepsilon_{1} r \\
& \leq \frac{1}{n} r=\frac{1}{n}\|\mathbf{x}\|
\end{aligned}
$$

which shows that

$$
\left\|\mathbf{T}_{\lambda} \mathbf{x}\right\|=\sum_{i=1}^{n} \sup _{t \in J}\left|\left(T_{\lambda}^{i} \mathbf{x}\right)(t)\right| \leq\|\mathbf{x}\|, \quad \forall \mathbf{x} \in \partial K_{r \theta} .
$$

If $f_{i}^{\infty}=0$, similar to the proof of (4.1), there exists $R>\rho$ such that $f_{i}(t, \mathbf{x}) \leq \varepsilon_{2}\|\mathbf{x}\|$, for $\|\mathbf{x}\| \geq R, t \in J$, where $\varepsilon_{2}>0$ satisfies $\lambda \varepsilon_{2}\left\|G_{i}\right\|_{q}\left\|g_{i}\right\|_{p} \leq \frac{1}{n}$, and, for $\mathbf{x} \in \partial K_{R \theta}$, we have

$$
\left\|\mathbf{T}_{\lambda} \mathbf{x}\right\| \leq\|\mathbf{x}\|
$$

On the other hand, from (ii), when a $\rho>0$ is fixed, then there exists a $\lambda_{0}>0$ such that $f_{i}(t, \mathbf{x}) \geq \delta>\frac{1}{\lambda}\left[\gamma^{*} \delta N_{i} \int_{\theta_{j}}^{1-\theta_{j}} G_{i}(s) d s\right]^{-1} \rho$ for $\lambda>\lambda_{0}, \mathbf{x} \in \partial K_{\rho \theta}$.

Therefore, for $\mathbf{x} \in \partial K_{\rho \theta}, t \in J$, we have

$$
\begin{aligned}
\left\|\mathbf{T}_{\lambda} \mathbf{x}\right\| & \geq \sup _{t \in J}\left|\left(T_{\lambda}^{i} \mathbf{x}\right)(t)\right| \\
& =\lambda \sup _{t \in J} \int_{0}^{1} G_{i}(t, s) g_{i}(s) f_{i}(s, \mathbf{x}(s)) d s \\
& \geq \min _{t \in\left[\theta_{j}, 1-\theta_{j}\right]} \lambda \delta \int_{0}^{1} G_{i}(t, s) g_{i}(s) d s \\
& \geq \gamma^{*} \lambda \delta N_{i} \int_{0}^{1} G_{i}(s) d s \\
& \geq \gamma^{*} \lambda \delta N_{i} \int_{\theta_{j}}^{1-\theta_{j}} G_{i}(s) d s \\
& >\rho=\|\mathbf{x}\| .
\end{aligned}
$$

Consequently, for $x \in \partial K_{\rho \theta}$, we have

$$
\left\|\mathbf{T}_{\lambda} \mathbf{x}\right\|>\|\mathbf{x}\|
$$

By Lemma 3.3, for all $\lambda>\lambda_{0},(6.1)$ and (6.3), (6.2) and (6.3), respectively, show that $\mathbf{T}_{\lambda}$ has a fixed point $\mathbf{x}^{*} \in \overline{\mathbf{K}}_{r, \rho}, r \leq\left\|\mathbf{x}^{*}\right\|<\rho$ and $\sum_{i=1}^{n} x_{i}^{*}(t) \geq \gamma\left\|\mathbf{x}^{*}\right\|, t \in[\theta, 1-\theta]$ or $\mathbf{x}^{*} \in \overline{\mathbf{K}}_{\rho, R}$, $\rho<\left\|\mathbf{x}^{*}\right\| \leq R$ and $\sum_{i=1}^{n} x_{i}^{*}(t) \geq \gamma\left\|x^{*}\right\|>0, t \in[\theta, 1-\theta]$. Thus it follows that system (1.1)(1.2) has at least one positive solution $\mathbf{x}^{*}$ for all $\lambda>\lambda_{0}$.

Theorem 6.4 Assume that $\left(H_{1}\right)$ and $\left(H_{3}\right)$ hold. Furthermore, lett the following two conditions hold:

(i) $f_{i}^{0}=0$ and $f_{i}^{\infty}=0$;

(ii) there exist $\rho>0, \delta>0$, such that $f_{i}(t, \mathbf{x}) \geq \delta$ for $\|\mathbf{x}\| \geq \rho, t \in J$, are satisfied, then there exists $\lambda_{0}>0$ such that, for all $\lambda>\lambda_{0}$, system (1.1)-(1.2) has at least two positive solutions $\mathbf{x}^{*}$ and $\mathbf{x}^{* *}$. 
Proof The proof is similar to that of Theorem 6.3. By Lemma 3.3, (6.1)-(6.3) show that $\mathbf{T}_{\lambda}$ has at least two fixed points $\mathbf{x}^{*}, \mathbf{x}^{* *}$, where $\mathbf{x}^{*} \in \overline{\mathbf{K}}_{r, \rho}, r \leq\left\|\mathbf{x}^{*}\right\|<\rho$ and $\sum_{i=1}^{n} x_{i}^{*}(t) \geq \gamma\left\|\mathbf{x}^{*}\right\|>$ $0, t \in[\theta, 1-\theta], \mathbf{x}^{* *} \in \overline{\mathbf{K}}_{\rho, R}, \rho<\left\|\mathbf{x}^{*}\right\| \leq R$ and $\sum_{i=1}^{n} x_{i}^{* *}(t) \geq \gamma\left\|\mathbf{x}^{* *}\right\|, t \in[\theta, 1-\theta]$. Thus it follows that system (1.1)-(1.2) has at least two positive solutions $\mathbf{x}^{*}, \mathbf{x}^{* *}$ for all $\lambda>\lambda_{0}$.

Remark 6.2 Results similar to Theorems 6.3-6.4 have been established by Wang [57] for other types of $n$-dimensional system.

Remark 6.3 Boundary value problem with infinitely many singularities has been studied by Kaufmann, Kosmatov, Wang and Feng. For more details on this study, we refer the reader to $[58,59]$ for a second order two point boundary value problem.

\section{An example}

In Example 7.1, we select $n=3, \alpha=\frac{5}{2}, a=2, b=1$.

Example 7.1 Consider the following system:

$$
\left\{\begin{array}{l}
\mathbf{D}_{0^{+}}^{\alpha} \mathbf{x}(t)+\lambda \mathbf{g}(t) \mathbf{f}(t, \mathbf{x}(t))=0, \quad 0<t<1 \\
\mathbf{x}(0)=\mathbf{x}^{\prime}(0)=0 \\
a \mathbf{x}(1)+b \mathbf{x}^{\prime}(1)=\int_{0}^{1} \mathbf{h}(t) \mathbf{x}(t) d t
\end{array}\right.
$$

where

$$
\begin{aligned}
& \mathbf{g}(t)=\left(\begin{array}{ccc}
g_{1}(t) & 0 & 0 \\
0 & g_{2}(t) & 0 \\
0 & 0 & g_{3}(t)
\end{array}\right), \quad \mathbf{h}(t)=\left(\begin{array}{lll}
5 & 0 & 0 \\
0 & 5 & 0 \\
0 & 0 & 5
\end{array}\right), \\
& \mathbf{f}(t, \mathbf{x})=\left(\begin{array}{l}
\left(\frac{1}{6}+\frac{1}{6} t\right) L x_{1}+\frac{1}{3} L x_{2}++\frac{1}{3} L x_{3} \\
\left(\frac{1}{6}+\frac{1}{6} t\right) L x_{1}+\frac{1}{3} L x_{2}++\frac{1}{3} L x_{3} \\
\left(\frac{1}{6}+\frac{1}{6} t\right) L x_{1}+\frac{1}{3} L x_{2}++\frac{1}{3} L x_{3}
\end{array}\right),
\end{aligned}
$$

where $L$ is defined in (4.1), $h_{i}(t) \equiv 5$ on $J, f_{i}(t, \mathbf{x})=f_{i}\left(t, x_{1}, x_{2}, x_{3}\right)=\left(\frac{1}{6}+\frac{1}{6} t\right) L x_{1}+\frac{1}{3} L x_{2}+$ $+\frac{1}{3} L x_{3}$ on $t \in J, g_{i}(t)(i=1,2,3)$ are singular at $t_{j}^{\prime}, j=1,2, \ldots$, and

$$
t_{j}^{\prime}=\frac{2}{5}-\frac{1}{10} \sum_{i=1}^{j} \frac{1}{(2 i-1)^{4}}, \quad j=1,2, \ldots
$$

It follows from (7.2) that

$$
\begin{aligned}
& t_{1}^{\prime}=\frac{2}{5}-\frac{1}{10}=\frac{3}{10}, \\
& t_{j}^{\prime}-t_{j+1}^{\prime}=\frac{1}{10(2 j+1)^{4}}, \quad j=1,2, \ldots,
\end{aligned}
$$

and from $\sum_{j=1}^{\infty} \frac{1}{(2 j-1)^{4}}=\frac{\pi^{4}}{96}$, there is

$$
t_{0}^{\prime}=\lim _{j \rightarrow \infty} t_{j}^{\prime}=\frac{2}{5}-\frac{1}{10} \sum_{j=1}^{\infty} \frac{1}{(2 j-1)^{4}}=\frac{2}{5}-\frac{1}{10} \cdot \frac{\pi^{4}}{96}=\frac{2}{5}-\frac{\pi^{4}}{960}>\frac{1}{10} .
$$




\section{Consider the function}

$$
g_{1}(t)=\sum_{j=1}^{\infty} g_{j}^{(1)}(t), \quad g_{2}(t)=\sum_{j=1}^{\infty} g_{j}^{(2)}(t), \quad g_{3}(t)=\sum_{j=1}^{\infty} g_{j}^{(3)}(t), \quad t \in J,
$$

where

$$
\begin{aligned}
& g_{j}^{(1)}(t)= \begin{cases}\frac{j+2}{(j+1) !\left(t_{j}^{\prime}+t_{j+1}^{\prime}\right)}, & t \in\left[0, \frac{t_{j}^{\prime}+t_{j+1}^{\prime}}{2}\right), \\
\frac{1}{\sqrt{t_{j}^{\prime}-t}}, & t \in\left[\frac{t_{j}^{\prime}+t_{j+1}^{\prime}}{2}, t_{j}^{\prime}\right), \\
\frac{1}{\sqrt{t-t_{j}^{\prime}},} & t \in\left(t_{j}^{\prime}, \frac{t_{j}^{\prime}+t_{j-1}^{\prime}}{2}\right], \\
\frac{j+2}{(j+1) !\left(2-t_{j}^{\prime}-t_{j-1}^{\prime}\right)}, & t \in\left(\frac{t_{j}^{\prime}+t_{j-1}^{\prime}}{2}, 1\right],\end{cases} \\
& g_{j}^{(2)}(t)= \begin{cases}\frac{2}{(2 j-2) !\left(t_{j}^{\prime}+t_{j+1}^{\prime}\right)}, & t \in\left[0, \frac{t_{j}^{\prime}+t_{j+1}^{\prime}}{2}\right), \\
\frac{1}{\sqrt{t_{j}^{\prime}-t}}, & t \in\left[\frac{t_{j}^{\prime}+t_{j+1}^{\prime}}{2}, t_{j}^{\prime}\right), \\
\frac{1}{\sqrt{t-t_{j}^{\prime}},} & t \in\left(t_{j}^{\prime}, \frac{t_{j}^{\prime}+t_{j-1}^{\prime}}{2}\right], \\
\frac{2}{(2 j-2) !\left(2-t_{j}^{\prime}-t_{j-1}^{\prime}\right)}, & t \in\left(\frac{t_{j}^{\prime}+t_{j-1}^{\prime}}{2}, 1\right],\end{cases} \\
& g_{j}^{(3)}(t)= \begin{cases}\frac{j}{2\left(t_{j}^{\prime}+t_{j+1}^{\prime}\right)}, & t \in\left[0, \frac{t_{j}^{\prime}+t_{j+1}^{\prime}}{2}\right), \\
\frac{1}{\sqrt{t_{j}^{\prime}-t}}, & t \in\left[\frac{t_{j}^{\prime}+t_{j+1}^{\prime}}{2}, t_{j}^{\prime}\right), \\
\frac{1}{\sqrt{t-t_{j}^{\prime}},} & t \in\left(t_{j}^{\prime}, \frac{t_{j}^{\prime}+t_{j-1}^{\prime}}{2}\right], \\
\frac{j}{2\left(2-t_{j}^{\prime}-t_{j-1}^{\prime}\right)}, & t \in\left(\frac{t_{j}^{\prime}+t_{j-1}^{\prime}}{2}, 1\right] .\end{cases}
\end{aligned}
$$

From $\sum_{j=1}^{\infty} \frac{j+2}{(j+1) !}=2 e-3, \sum_{j=1}^{\infty} \frac{2}{(2 j-2) !}=e+e^{-1}, \sum_{j=1}^{\infty} \frac{j}{2 j}=2$ and $\sum_{j=1}^{\infty} \frac{1}{(2 j-1)^{2}}=\frac{\pi^{2}}{8}$, we get

$$
\begin{aligned}
\sum_{j=1}^{\infty} \int_{0}^{1} g_{j}^{(1)}(t) d t= & \sum_{j=1}^{\infty}\left\{\int_{0}^{\frac{t_{j}^{\prime}+t_{j+1}^{\prime}}{2}} \frac{j+2}{(j+1) !\left(t_{j}^{\prime}+t_{j+1}^{\prime}\right)} d t+\int_{\frac{t_{j-1}^{\prime}+t_{j}^{\prime}}{2}}^{1} \frac{j+2}{(j+1) !\left(2-t_{j}^{\prime}-t_{j-1}^{\prime}\right)} d t\right. \\
& \left.+\int_{\frac{t_{j}^{\prime}+t_{j+1}^{\prime}}{2}}^{t_{j}^{\prime}} \frac{1}{\sqrt{t_{j}^{\prime}-t}} d t+\int_{t_{j}^{\prime}}^{\frac{t_{j-1}^{\prime}+t_{j}^{\prime}}{2}} \frac{1}{\sqrt{t-t_{j}^{\prime}}} d t\right\} \\
= & \sum_{j=1}^{\infty} \frac{j+2}{(j+1) !}+\sqrt{2} \sum_{j=1}^{\infty}\left(\sqrt{t_{j}^{\prime}-t_{j+1}^{\prime}}+\sqrt{t_{j-1}^{\prime}-t_{j}^{\prime}}\right) \\
= & 2 e-3+\frac{\sqrt{2}}{\sqrt{10}} \sum_{j=1}^{\infty}\left(\frac{1}{(2 j+1)^{2}}+\frac{1}{(2 j-1)^{2}}\right) \\
= & 2 e-3+\frac{\sqrt{2}}{\sqrt{10}}\left(\frac{\pi^{2}}{8}-1+\frac{\pi^{2}}{8}\right) \\
= & 2 e-3+\frac{\sqrt{2}}{\sqrt{10}}\left(\frac{\pi^{2}}{4}-1\right)
\end{aligned}
$$




$$
\begin{aligned}
& \sum_{j=1}^{\infty} \int_{0}^{1} g_{j}^{(2)}(t) d t=\sum_{j=1}^{\infty}\left\{\int_{0}^{\frac{t_{j}^{\prime}+t_{j+1}^{\prime}}{2}} \frac{2}{(2 j-2) !\left(t_{j}^{\prime}+t_{j+1}^{\prime}\right)} d t+\int_{\frac{t_{-1}^{\prime}+t_{j}^{\prime}}{2}}^{1} \frac{2}{(2 j-2) !\left(2-t_{j}^{\prime}-t_{j-1}^{\prime}\right)} d t\right. \\
& \left.+\int_{\frac{t_{j}^{\prime}+t_{j+1}^{\prime}}{2}}^{t_{j}^{\prime}} \frac{1}{\sqrt{t_{j}^{\prime}-t}} d t+\int_{t_{j}^{\prime}}^{\frac{t_{j-1}^{\prime}+t_{j}^{\prime}}{2}} \frac{1}{\sqrt{t-t_{j}^{\prime}}} d t\right\} \\
& =\sum_{j=1}^{\infty} \frac{2}{(2 j-2) !}+\sqrt{2} \sum_{j=1}^{\infty}\left(\sqrt{t_{j}^{\prime}-t_{j+1}^{\prime}}+\sqrt{t_{j-1}^{\prime}-t_{j}^{\prime}}\right) \\
& =e+e^{-1}+\frac{\sqrt{2}}{\sqrt{10}} \sum_{j=1}^{\infty}\left(\frac{1}{(2 j+1)^{2}}+\frac{1}{(2 j-1)^{2}}\right) \\
& =e+e^{-1}+\frac{\sqrt{2}}{\sqrt{10}}\left(\frac{\pi^{2}}{8}-1+\frac{\pi^{2}}{8}\right) \\
& =e+e^{-1}+\frac{\sqrt{2}}{\sqrt{10}}\left(\frac{\pi^{2}}{4}-1\right) \\
& \sum_{j=1}^{\infty} \int_{0}^{1} g_{j}^{(3)}(t) d t=\sum_{j=1}^{\infty}\left\{\int_{0}^{\left(t_{j}^{\prime}+t_{j+1}^{\prime}\right) / 2} \frac{j}{2^{j\left(t_{n}^{\prime}+t_{j+1}^{\prime}\right)}} d t+\int_{\left(t_{j-1}^{\prime}+t_{j}^{\prime}\right) / 2}^{1} \frac{j}{2 j\left(2-t_{j}^{\prime}-t_{j-1}^{\prime}\right)} d t\right. \\
& \left.+\int_{\left(t_{j}^{\prime}+t_{j+1}^{\prime}\right) / 2}^{t_{j}^{\prime}} \frac{1}{\sqrt{t_{j}^{\prime}-t}} d t+\int_{t_{j}^{\prime}}^{\left(t_{j-1}^{\prime}+t_{j}^{\prime}\right) / 2} \frac{1}{\sqrt{t-t_{j}^{\prime}}} d t\right\} \\
& =\sum_{j=1}^{\infty} \frac{j}{2^{j}}+\sqrt{2} \sum_{j=1}^{\infty}\left(\sqrt{t_{j}^{\prime}-t_{j+1}^{\prime}}+\sqrt{t_{j-1}^{\prime}-t_{j}^{\prime}}\right) \\
& =2+\frac{\sqrt{2}}{\sqrt{10}} \sum_{j=1}^{\infty}\left(\frac{1}{(2 j+1)^{2}}+\frac{1}{(2 j-1)^{2}}\right) \\
& =2+\frac{\sqrt{2}}{\sqrt{10}}\left(\frac{\pi^{2}}{8}-1+\frac{\pi^{2}}{8}\right) \\
& =2+\frac{\sqrt{2}}{\sqrt{10}}\left(\frac{\pi^{2}}{4}-1\right) \text {. }
\end{aligned}
$$

Thus, from (7.3), (7.4) and (7.5), it is easy to see that

$$
\begin{aligned}
& \int_{0}^{1} g_{1}(t) d t=\int_{0}^{1} \sum_{j=1}^{\infty} g_{j}^{(1)}(t) d t=\sum_{j=1}^{\infty} \int_{0}^{1} g_{j}^{(1)}(t) d t=2 e-3+\frac{\sqrt{2}}{\sqrt{10}}\left(\frac{\pi^{2}}{4}-1\right)<+\infty \\
& \int_{0}^{1} g_{2}(t) d t=\int_{0}^{1} \sum_{j=1}^{\infty} g_{j}^{(2)}(t) d t=\sum_{j=1}^{\infty} \int_{0}^{1} g_{j}^{(2)}(t) d t=e+e^{-1}+\frac{\sqrt{2}}{\sqrt{10}}\left(\frac{\pi^{2}}{4}-1\right)<+\infty \\
& \int_{0}^{1} g_{3}(t) d t=\int_{0}^{1} \sum_{j=1}^{\infty} g_{j}^{(3)}(t) d t=\sum_{j=1}^{\infty} \int_{0}^{1} g_{j}^{(3)}(t) d t=2+\frac{\sqrt{2}}{\sqrt{10}}\left(\frac{\pi^{2}}{4}-1\right)<+\infty
\end{aligned}
$$

Therefore $g_{i}(t) \in L^{1}[0,1], i=1,2,3$, which shows that conditions $\left(H_{1}\right)$ and $\left(H_{2}\right)$ hold. 
On the other hand, it is not difficult to see that by means of the definition of $\mathbf{f}(t, \mathbf{x})$ condition $\left(H_{3}\right)$ holds. At the same time, it follows from $a=2, b=1, \alpha=\frac{5}{2}$ and $h_{i}(t) \equiv 5$ that

$$
\mu_{i}=\int_{0}^{1} h_{i}(t) t^{\alpha-1} d t=2<a+b(\alpha-1)=\frac{7}{2}, \quad i=1,2,3,
$$

which shows that condition $\left(H_{4}\right)$ holds.

Next, we show that conditions $\left(H_{5}\right)$ and $\left(H_{6}\right)$ of Theorem 4.1 hold. In fact, for any $t \in J$, $\|\mathbf{x}\| \in\left[0, R_{j}\right]$, we get

$$
f_{i}(t, \mathbf{x})=\left(\frac{1}{6}+\frac{1}{6} t\right) L x_{1}+\frac{1}{3} L x_{2}++\frac{1}{3} L x_{3} \leq L R_{j}
$$

for any $t \in\left[\theta_{j}, 1-\theta_{j}\right],\|\mathbf{x}\| \in\left[\gamma_{j}^{*} r_{j}, r_{j}\right]$, we get

$$
f_{i}(t, \mathbf{x})=\left(\frac{1}{6}+\frac{1}{6} t\right) L x_{1}+\frac{1}{3} L x_{2}++\frac{1}{3} L x_{3} \geq l r_{j}, \quad \text { where } l=\left(\frac{1}{3} L \theta_{j} \gamma_{j}^{*}+\frac{1}{3} L+\frac{1}{3} L\right)
$$

Therefore, it follows from Theorem 4.1 that there exists $\lambda_{0}>0$, such that, for $\lambda>\lambda_{0}$, system (7.1) has denumerably many positive solutions $\left\{\mathbf{x}_{j}\right\}_{j=1}^{\infty}$ satisfying

$$
r_{j} \leq\left\|\mathbf{x}_{j}\right\| \leq R_{j}, \quad j=1,2, \ldots
$$

\section{Acknowledgements}

The authors are grateful to anonymous referees for their constructive comments and suggestions, which have greatly improved this paper.

\section{Funding}

This work is sponsored by the National Natural Science Foundation of China (11401031), the Beijing Natural Science Foundation (1163007), the Scientific Research Project of Construction for Scientific and Technological Innovation Service Capacity (KM201611232017) and the teaching reform project of Beijing Information Science \& Technology University (2018JGZD41).

\section{Availability of data and materials}

Not applicable.

Ethics approval and consent to participate

Not applicable.

\section{Competing interests}

The authors declare that there is no conflict of interest regarding the publication of this manuscript. The authors declare that they have no competing interests.

\section{Consent for publication}

Not applicable.

\section{Authors' contributions}

The authors contributed equally in this article. They have all read and approved the final manuscript.

\section{Publisher's Note}

Springer Nature remains neutral with regard to jurisdictional claims in published maps and institutional affiliations.

Received: 8 December 2017 Accepted: 16 April 2018 Published online: 23 April 2018

\section{References}

1. Miller, K.S., Ross, B.: An Introduction to the Fractional Calculus and Fractional Differential Equations. Wiley, New York (1993)

2. Samko, G., Kilbas, A.A., Marichev, O.I.: Fractional Integrals and Derivatives: Theory and Applications. Gordon \& Breach, Yverdon (1993) 
3. Podlubny, I.: Fractional Differential Equations. Mathematics in Sciences and Engineering, vol. 198. Academic Press, San Diego (1999)

4. Lakshmikantham, V., Leela, S., Vasundhara Devi, J.: Theory of Fractional Dynamic Systems. Cambridge Academic, Cambridge (2009)

5. Guan, Y., Zhao, Z., Lin, X.: On the existence of positive solutions and negative solutions of singular fractional differential equations via global bifurcation techniques. Bound. Value Probl. 2016, 141 (2016)

6. Guo, L., Liu, L., Wu, Y:: Existence of positive solutions for singular fractional differential equations with infinite-point boundary conditions. Nonlinear Anal., Model. Control 21, 635-650 (2016)

7. Bai, Z., Lü, H.: Positive solutions of boundary value problems of nonlinear fractional differential equation. J. Math. Anal. Appl. 311, 495-505 (2005)

8. Feng, M., Zhang, X., Ge, W.: New existence results for higher-order nonlinear fractional differential equation with integral boundary conditions. Bound. Value Probl. 2011, 720702 (2011)

9. Zhang, X., Liu, L., Wiwatanapataphee, B., Wu, Y.: The eigenvalue for a class of singular $p$-Laplacian fractional differential equations involving the Riemann-Stieltjes integral boundary condition. Appl. Math. Comput. 235, 412-422 (2014)

10. Bai, Z., Sun, W.: Existence and multiplicity of positive solutions for singular fractional boundary value problems. Comput. Math. Appl. 63, 1369-1381 (2012)

11. Ahmad, B., Nieto, J.J.: Existence results for nonlinear boundary value problems of fractional integrodifferential equations with integral boundary conditions. Bound. Value Probl. 2009, 708576 (2009)

12. Bai, Z: On positive solutions of a nonlocal fractional boundary value problem. Nonlinear Anal. 72, 916-924 (2010)

13. Wang, Y., Liu, L., Zhang, X., Wu, Y.: Positive solutions of a fractional semipositone differential system arising from the study of HIV infection models. Appl. Math. Comput. 258, 312-324 (2015)

14. Liu, X., Jia, M., Ge, W.: The method of lower and upper solutions for mixed fractional four-point boundary value problem with p-Laplacian operator. Appl. Math. Lett. 65, 56-62 (2017)

15. Hao, X.: Positive solution for singular fractional differential equations involving derivatives. Adv. Differ. Equ. 2016, 139 (2016)

16. Wang, Y., Liu, L., Wu, Y.: Positive solutions for a nonlocal fractional differential equation. Nonlinear Anal. 74, 3599-3605 (2011)

17. Zhang, $X ., L i u, L ., W u, Y:$ The eigenvalue problem for a singular higher order fractional differential equation involving fractional derivatives. Appl. Math. Comput. 218, 8526-8536 (2012)

18. Salem, H.A.H.: On the fractional order $m$-point boundary value problem in reflexive Banach spaces and weak topologies. J. Comput. Appl. Math. 224, 565-572 (2009)

19. Kaufmann, E.R., Mboumi, E.: Positive solutions of a boundary value problem for a nonlinear fractional differential equation. Electron. J. Qual. Theory Differ. Equ. 2008, 3 (2008)

20. Günendi, M., Yaslan, I.: Positive solutions of higher-order nonlinear multi-point fractional equations with integral boundary conditions. Fract. Calc. Appl. Anal. 19, 989-1009 (2016)

21. Graef, J.R., Kong, L., Kong, Q., Wang, M.: Uniqueness of positive solutions of fractional boundary value problems with non-homogeneous integral boundary conditions. Fract. Calc. Appl. Anal. 15, 509-528 (2012)

22. Jiang, J., Liu, L., Wu, Y.: Positive solutions for second-order differential equations with integral boundary conditions. Bull. Malays. Math. Sci. Soc. 37, 779-796 (2014)

23. Cabada, A., Wang, G.: Positive solutions of nonlinear fractional differential equations with integral boundary value conditions. J. Math. Anal. Appl. 389, 403-411 (2012)

24. Alsulami, H.H., Ntouyas, S.K., Agarwal, R.P., Ahmad, B., Alsaedi, A.: A study of fractional-order coupled systems with a new concept of coupled non-separated boundary conditions. Bound. Value Probl. 2017, 11 (2017)

25. Sokolov, I.M., Klafter, J., Blumen, A.: Fractional kinetics. Phys. Today 55, 48-54 (2002)

26. Petras, I., Magin, R.L.: Simulation of drug uptake in a two compartmental fractional model for a biological system. Commun. Nonlinear Sci. Numer. Simul. 16, 4588-4595 (2011)

27. Ding, Y., Wang, Z., Ye, H.: Optimal control of a fractional-order HIV-immune system with memory. IEEE Trans. Control Syst. Technol. 20, 763-769 (2012)

28. Carvalho, A., Pinto, C.M.A.: A delay fractional order model for the co-infection of malaria and HIV/AIDS. Int. J. Dyn. Control 5, 168-186 (2017)

29. Javidi, M., Ahmad, B.: Dynamic analysis of time fractional order phytoplankton-toxic phytoplankton-zooplankton system. Ecol. Model. 318, 8-18 (2015)

30. Ge, Z.M., Ou, C.Y: Chaos synchronization of fractional order modified Duffing systems with parameters excited by a chaotic signal. Chaos Solitons Fractals 35, 705-717 (2008)

31. Faieghi, M., Kuntanapreeda, S., Delavari, H., Baleanu, D.: LMI-based stabilization of a class of fractional-order chaotic systems. Nonlinear Dyn. 72, 301-309 (2013)

32. Zhang, F., Chen, G., Li, C., Kurths, J.: Chaos synchronization in fractional differential systems. Philos. Trans. R. Soc. Lond. A 371, 20120155 (2013)

33. Senol, B., Yeroglu, C.: Frequency boundary of fractional order systems with nonlinear uncertainties. J. Franklin Inst. 350, 1908-1925 (2013)

34. Henderson, J., Luca, R.: Nonexistence of positive solutions for a system of coupled fractional boundary value problems. Bound. Value Probl. 2015, 138 (2015)

35. Ahmad, B., Ntouyas, S.K.: Existence results for a coupled system of Caputo type sequential fractional differential equations with nonlocal integral boundary conditions. Appl. Math. Comput. 266, 615-622 (2015)

36. Wang, J., Zhang, Y.: Analysis of fractional order differential coupled systems. Math. Methods Appl. Sci. 38, 3322-3338 (2015)

37. Tariboon, J., Ntouyas, S.K., Sudsutad, W.: Coupled systems of Riemann-Liouville fractional differential equations with Hadamard fractional integral boundary conditions. J. Nonlinear Sci. Appl. 9, 295-308 (2016)

38. Ahmad, B., Ntouyas, S.K., Alsaedi, A.: On a coupled system of fractional differential equations with coupled nonlocal and integral boundary conditions. Chaos Solitons Fractals 83, 234-241 (2016)

39. Han, X., Yang, X.: Existence and multiplicity of positive solutions for a system of fractional differential equation with parameters. Bound. Value Probl. 2017, 78 (2017) 
40. Su, X: Boundary value problem for a coupled system of nonlinear fractional differential equations. Appl. Math. Lett. $22,64-69(2009)$

41. Shah, K., Khan, R.A.: Existence and uniqueness of positive solutions to a coupled system of nonlinear fractional order differential equations with anti periodic boundary conditions. Differ. Equ. Appl. 7, 245-262 (2015)

42. Yang, A., Ge, W.: Positive solutions for boundary value problems of $\mathrm{N}$-dimension nonlinear fractional differential system. Bound. Value Probl. 2008, 437453 (2008)

43. Benmezaï, A., Saadi, A.: Positive solutions for boundary value problems of $\mathrm{N}$-dimension nonlinear fractional differential system with integral boundary conditions. Fract. Differ. Calc. 7, 185-197 (2017)

44. Boucherif, A.: Second-order boundary value problems with integral boundary conditions. Nonlinear Anal. 70, 364-371 (2009)

45. Zhang, X., Feng, M., Ge, W.: Existence result of second-order differential equations with integral boundary conditions at resonance. J. Math. Anal. Appl. 353, 311-319 (2009)

46. Zhang, X., Ge, W.: Symmetric positive solutions of boundary value problems with integral boundary conditions. Appl. Math. Comput. 219, 3553-3564 (2012)

47. Hao, X., Liu, L., Wu, Y.: Positive solutions for second order impulsive differential equations with integral boundary conditions. Commun. Nonlinear Sci. Numer. Simul. 16, 101-111 (2011)

48. Jiang, J., Liu, L., Wu, Y.: Positive solutions for second order impulsive differential equations with Stieltjes integral boundary conditions. Adv. Differ. Equ. 2012, 124 (2012)

49. Jiang, J., Liu, L., Wu, Y.: Second-order nonlinear singular Sturm-Liouville problems with integral boundary problems. Appl. Math. Comput. 215, 1573-1582 (2009)

50. Kong, L.: Second order singular boundary value problems with integral boundary conditions. Nonlinear Anal. 72 , 2628-2638 (2010)

51. Feng, M., Ji, D., Ge, W.: Positive solutions for a class of boundary value problem with integral boundary conditions in Banach spaces. J. Comput. Appl. Math. 222, 351-363 (2008)

52. Ahmad, B., Alsaedi, A.: Existence of approximate solutions of the forced Duffing equation with discontinuous type integral boundary conditions. Nonlinear Anal., Real World Appl. 10, 358-367 (2009)

53. Mao, J., Zhao, Z:: The existence and uniqueness of positive solutions for integral boundary value problems. Bull. Malays. Math. Sci. Soc. 34, 153-164 (2011)

54. Liu, L., Hao, X., Wu, Y.: Positive solutions for singular second order differential equations with integral boundary conditions. Math. Comput. Model. 57, 836-847 (2013)

55. Liu, L., Li, H., Liu, C., Wu, Y.: Existence and uniqueness of positive solutions for singular fractional differential systems with coupled integral boundary value problems. J. Nonlinear Sci. Appl. 10, 243-262 (2017)

56. Guo, D. Lakshmikantham, V.: Nonlinear Problems in Abstract Cones. Academic Press, New York (1988)

57. Wang, H.: On the number of positive solutions of nonlinear systems. J. Math. Anal. Appl. 281, 287-306 (2003)

58. Kaufmann, E.R., Kosmatov, N.: A multiplicity result for a boundary value problem with infinitely many singularities. J. Math. Anal. Appl. 269, 444-453 (2002)

59. Wang, M., Feng, M.: Infinitely many singularities and denumerably many positive solutions for a second-order impulsive Neumann boundary value problem. Bound. Value Probl. 2017, 50 (2017)

\section{Submit your manuscript to a SpringerOpen ${ }^{\circ}$ journal and benefit from:}

- Convenient online submission

- Rigorous peer review

- Open access: articles freely available online

- High visibility within the field

- Retaining the copyright to your article

Submit your next manuscript at $\gg$ springeropen.com 